\title{
Identifying the Impact of Intimate Partner Violence in Humanitarian Settings: Using an Ecological Framework to Review 15 Years of Evidence
}

\author{
Melissa Meinhart ${ }^{1}$, Ilana Seff ${ }^{1}$, Katrina Troy ${ }^{1}$, Samantha McNelly ${ }^{1}$, Luissa Vahedi ${ }^{1}$ (D), Catherine Poulton ${ }^{2}$ \\ and Lindsay Stark 1 ,*
}

1 Brown School of Social Work, Washington University, in St. Louis 1 Brookings Drive, St. Louis, MO 63130, USA; melissa.m.meinhart@gmail.com (M.M.); seff@wustl.edu (I.S.); mskatrinatroy@gmail.com (K.T.); mcnelly.s@wustl.edu (S.M.); l.vahedi@wustl.edu (L.V.)

2 UNICEF, United Nations Plaza, New York, NY 10017, USA; cpoulton@unicef.org

* Correspondence: lindsaystark@wustl.edu; Tel.: +1-314-935-2219

check for updates

Citation: Meinhart, M.; Seff, I.; Troy, K.; McNelly, S.; Vahedi, L.; Poulton, C.; Stark, L. Identifying the Impact of Intimate Partner Violence in Humanitarian Settings: Using an Ecological Framework to Review 15 Years of Evidence. Int. J. Environ. Res. Public Health 2021, 18, 6963. https:/ / doi.org/10.3390/ijerph18136963

Academic Editor: Carol Cunradi

Received: 11 May 2021

Accepted: 7 June 2021

Published: 29 June 2021

Publisher's Note: MDPI stays neutral with regard to jurisdictional claims in published maps and institutional affiliations.

Copyright: (c) 2021 by the authors. Licensee MDPI, Basel, Switzerland. This article is an open access article distributed under the terms and conditions of the Creative Commons Attribution (CC BY) license (https:/ / creativecommons.org/licenses/by/ $4.0 /)$.

\begin{abstract}
Intimate partner violence (IPV) is a pervasive form of gender-based violence that exacerbates in humanitarian settings. This systematic review examined the myriad IPV impacts and the quality of existing evidence of IPV in humanitarian settings. Following the Preferred Reporting Items for Systematic Reviews and Meta-Analyses (PRISMA) procedures, a total of 51 articles were included from the 3924 screened. We identified the impact of IPV across two levels of the ecological framework: individual and microsystem. Our findings corroborated previous evidence that indicated IPV to be associated with adverse physical and mental health for survivors. Our findings also uniquely synthesized the intergenerational impact of IPV in humanitarian settings. However, findings highlighted a glaring gap in evidence examining the non-health impact of IPV for survivors in humanitarian settings and across levels of the ecological framework. Without enhanced research of women and girls and the violence they experience, humanitarian responses will continue to underachieve, and the needs of women and girls will continue to be relegated as secondary interests. Investment should prioritize addressing the range of both health and non-health impacts of IPV among individuals, families, and communities, as well as consider how the humanitarian environment influences these linkages.
\end{abstract}

Keywords: intimate partner violence; gender-based violence; humanitarian settings; ecological frameworks; systematic review

\section{Introduction}

Intimate Partner Violence (IPV) is a critically concerning form of gender-based violence in humanitarian settings. IPV includes violence that occurs within an intimate relationship of romantic partners or ex-partners, whether cohabitating or not [1]. Moreover, IPV can be exhibited through physical, sexual, or psychological harm and includes physical aggression, sexual coercion, psychological abuse, and controlling behaviors [2]. Violence perpetrated by intimate partners within the private sphere of the home continues to be an insidious form of gender-based violence during periods of acute crisis as well as protracted emergencies and post-conflict settings [3-7]. In humanitarian settings, known predictors of interpersonal violence within the household, including IPV, include conflict exposure, substance use, low economic status, adverse mental health, and limited social support [8], and many known determinants of IPV are exacerbated in emergency settings [9].

While less is known regarding the widespread impacts of IPV in humanitarian settings specifically, several reviews have synthesized evidence examining the global impacts of IPV on survivors. One review, not specific to low- and middle-income countries (LMICs) nor humanitarian settings, identified consistent evidence that IPV affected sexual risk-taking 
behaviors, risk of lifetime sexually transmitted infections (STIs), unwanted pregnancy or induced abortion, and sexual dysfunction [10]. Another review examining both physical and mental health consequences found that IPV survivors were more likely to exhibit physical injury, chronic pain, gastrointestinal issues, gynecological disorders, depression, and posttraumatic stress disorder (PTSD) [11]. Systematic reviews of violence against women and girls in LMICs found that IPV was associated with composite measures of adverse mental health, suicidal ideation and behavior, and symptoms of depression, posttraumatic stress, and disordered eating [12], as well as STIs, unwanted pregnancy or induced abortion, and number of sexual partners [13]. While these four reviews bolster our understanding of the consequences of experiencing IPV, they have three notable gaps: (1) these reviews did not consider IPV in humanitarian settings, (2) these reviews did not include non-health correlates with IPV, and (3) these reviews did not examine the impacts of IPV beyond the survivor. The importance of addressing each of these gaps is described below.

Humanitarian crises are associated with periods of extreme chronic stress, loss of health and social service infrastructure, and strained social support networks; all can serve to exacerbate the impacts of IPV $[14,15]$. Moreover, this fundamental breakdown within humanitarian settings could contribute to unique consequences of IPV when compared to non-humanitarian settings. For example, the socioeconomic precarity within humanitarian settings may not only contribute to elevated prevalence of IPV but may also limit survivors' abilities to return to or search for employment after experiencing IPV. Data collection of IPV in humanitarian settings is also difficult due to the instability inherent to natural disasters, infectious disease outbreaks, mass displacement, and civil unrest $[6,16,17]$. These measurement challenges have contributed to a paucity of IPV research in humanitarian settings; thus, little is known about how IPV impacts compare to those encountered in non-humanitarian settings.

Survivors in humanitarian settings may experience different types and severity of health impacts given the contextual variances between humanitarian and non-humanitarian settings. For example, women who would have otherwise accessed healthcare facilities for physical injuries from IPV may be unable to do so during a natural disaster, thus, increasing their risk for secondary infection or long-term disability. Moreover, research has yet to synthesize the non-health related impacts of IPV for survivors despite a growing body of evidence that has highlighted adverse economic outcomes for IPV survivors [18-21]. Other potential non-health impacts of IPV for survivors include loss of education and opportunity, productivity loss at work and home, stigma and shame, as well as diminished social capital, autonomy, and decision making.

Given the burden of violence placed on survivors, individual-level consequences of IPV are important to identify; however, IPV also has implications for the family unit and society. For example, witnessing IPV as a child has been linked to adverse mental health as well as an increased likelihood of perpetrating and experiencing IPV later in life [22-24]. IPV can also have harder-to-measure consequences at the community and societal levels. Women and girls who have experienced IPV may become further embedded in a cycle of victimization that perpetuates the feminization of poverty and the wider erosion of women's sexual and reproductive agency. Gender-based violence, including IPV, has also been demonstrated to have harmful macroeconomic impacts in non-humanitarian settings [25-30]. Other potential impacts across the ecological framework include intra-household tensions and poverty, increased service needs and strains (health, legal, housing, justice, etc.), as well as perpetuation of harmful social norms, structural violence, and gender inequities.

Thus, it is critically important to examine the breadth of IPV impacts in humanitarian settings to understand the complexity of IPV and inform response efforts. Comprehensively examining IPV can enable the critical examination across and between all levels of society. Heise's [31] seminal ecological framework illustrated the synergies between personal, situational, and sociocultural risk factors of male-perpetrated IPV across four levels: individual (i.e., IPV survivors and victims), microsystem (i.e., interpersonal interactions and relationships), exosystem (i.e., social and organizational structures), and 
macrosystem (i.e., influence on society). This risk framework has been adapted to humanitarian settings [9] and integrated for program design [32]; however, it has not been adapted as an impact framework. Building from the IPV and humanitarian literature, we adapted Heise's ecological framework to hypothesize the myriad impacts of IPV in humanitarian settings. The adapted framework in Figure 1 guided the expansive interest of this systematic review by mapping potential impacts of IPV in humanitarian settings at the individual, microsystem, exosystem, and macrosystem levels.

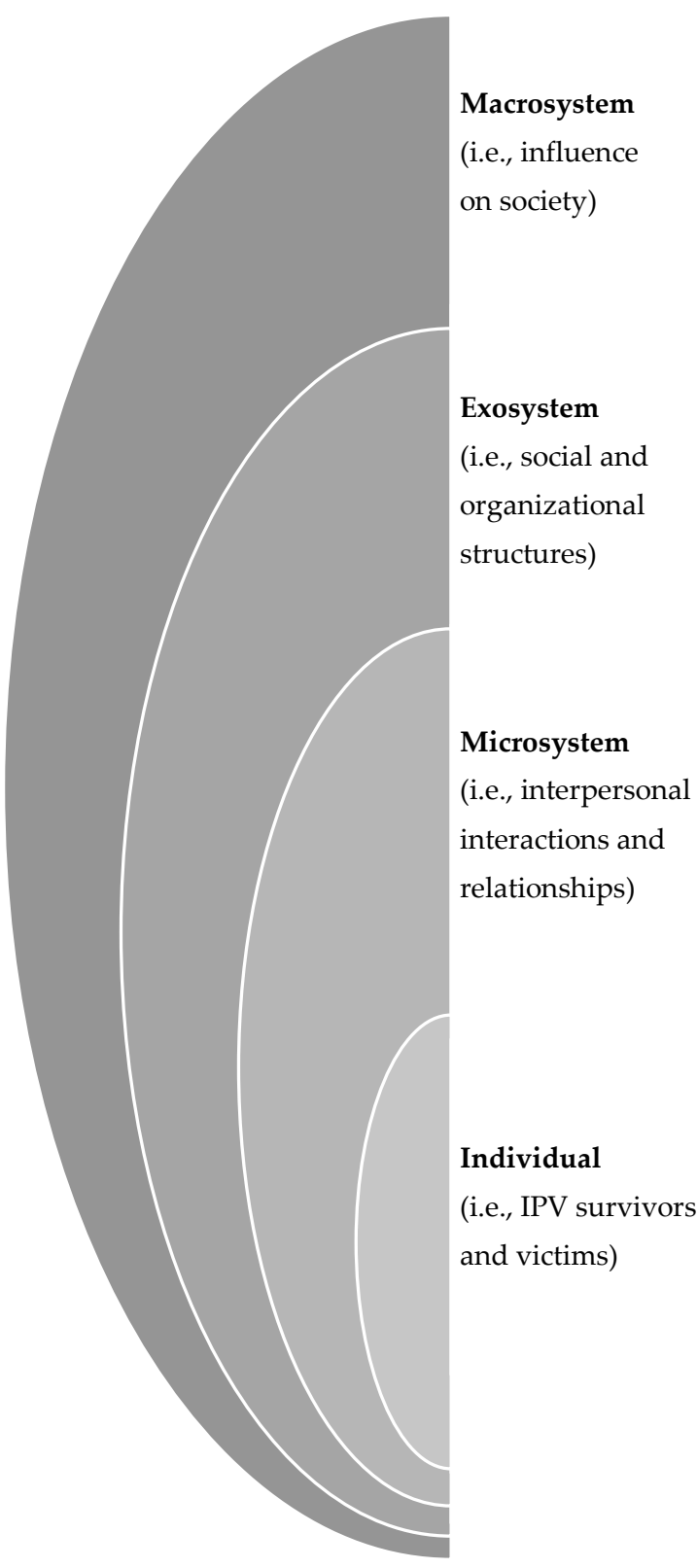

Perpetuation of patriarchal norms; exacerbated gender inequities; reduced national gross domestic product; stratification of socioeconomic standings between men and women; magnified violence prevalence; reduced opportunities for women and girls; elevated acceptance of intimate partner violence and gender based violence; magnified stigma.

Elevated community prevalence of violence (incl. intimate partner violence); increased service needs (health, legal, housing, justice, etc.); increased service provision and provider time costs; increased strain and stress on service providers and community members.

Increased domestic violence; relational tensions and negligence; household food insecurity \& nutrition; exacerbated intra-household poverty; children schooling (incl. performance, missed days, and truancy); children's overall physical health (incl. mortality for children under 5); children's mental health; children's criminality \& violence (incl. IPV) into adulthood; children behavioral and emotional functioning into adulthood.

Physical injury (bruises/cuts, broken bones, concussions, hemorrhaging); sexual and reproductive health (incl. HIV/STIs, unintended pregnancy, and abortion); out-ofpocket cost for service utilization (incl. health, legal, housing, justice); premature mortality (incl. maternal mortality \& suicide); loss of income and education; reduced number of days worked or unemployment; substance use; diminished social capital, autonomy and decision making; productivity loss (home/work); shame; mental health symptomology.

Figure 1. Impacts of intimate partner violence in humanitarian settings using an amended ecological framework.

Using the adapted ecological framework, this systematic review endeavored to examine the impact of IPV for survivors, families, communities, and society. Given the methodological challenges of collecting data in humanitarian settings, this review also examined the quality of studies from which the evidence was drawn. Understanding the full range of impacts that IPV has in humanitarian settings can simultaneously support critical programming for survivors and address the needs of families and communities affected by IPV. 


\section{Methods}

\subsection{Search Strategy}

We conducted a systematic review of peer-reviewed articles that examined the impacts of IPV in humanitarian settings using the Preferred Reporting Items for Systematic Reviews and Meta-Analyses (PRISMA) guidelines [33]. Aligning with standardized violence definitions brokered by the WHO Multi-Country Study in 2005 on Women's Health and Domestic Violence against Women [34], the search included articles published between 2005 and 2020. Our search terms strategy was applied to four databases: Embase through Elsevier, Medline via EBSCO, PsycInfo through Ebscohost, and Scopus via Scopus. Abstracts from the identified articles $(\mathrm{N}=3924)$ were imported into Covidence for duplication removal, abstract review, and full-text review. All abstracts and full texts were reviewed by the authors to determine if they met review criteria at each stage. Conflicts at both the abstract and full-text review stages were reread and discussed by the authors for the final decision. A visual representation of the review process is available in Figure 2.

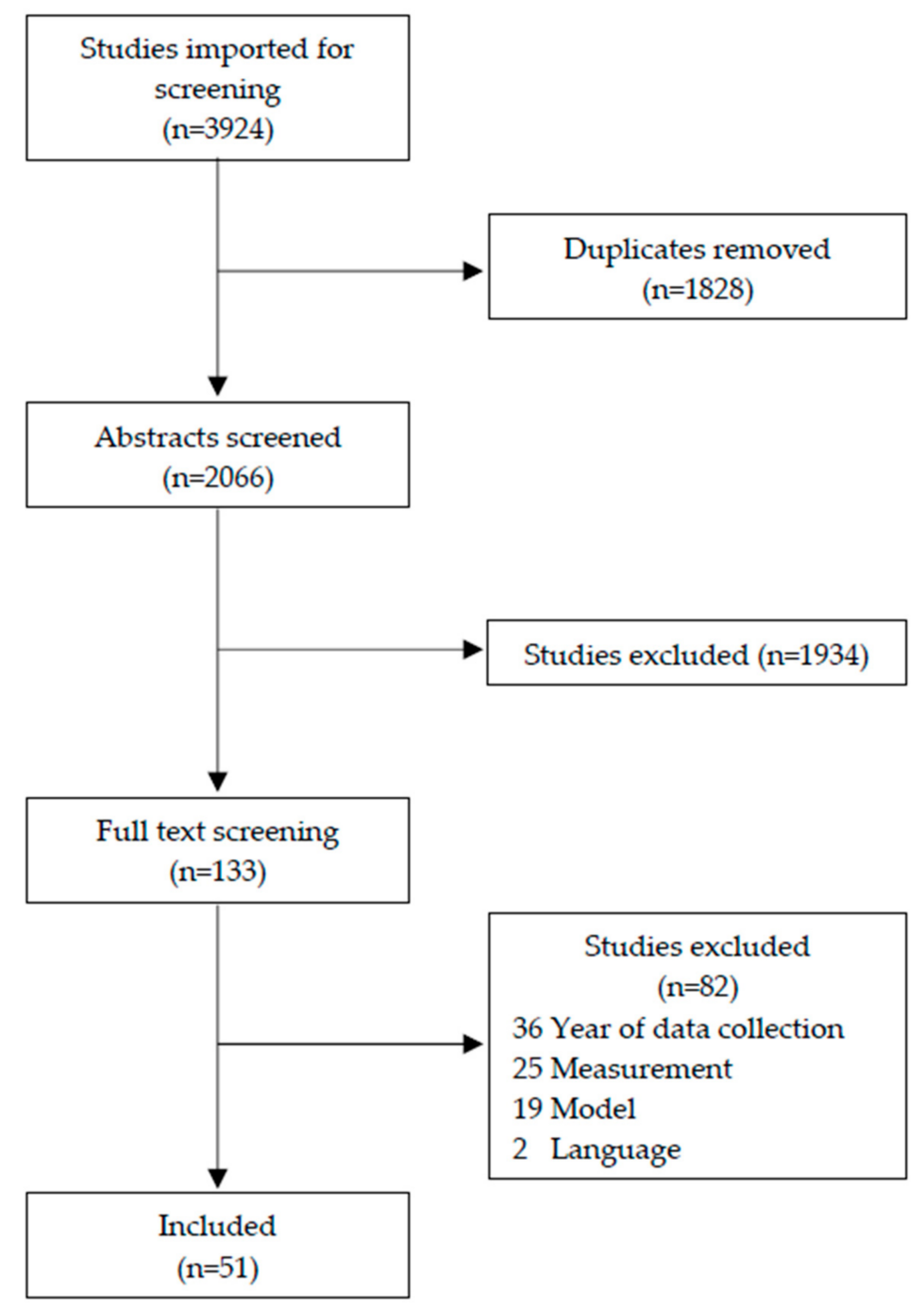

Figure 2. PRISMA flow diagram.

\subsection{Inclusion and Exclusion Criteria}

Using systematic review software (Covidence), abstracts were reviewed per inclusion criteria. Criteria included abstracts that utilized quantitative methods and abstracts that included at least one form of IPV as an independent variable. Examples of eligible IPV variables included experiencing physical IPV during adulthood, mean community 
rates of IPV, and witnessing intra-parental violence during childhood. Using the United Nation's Financial Tracking System (FTS) [35], articles were considered eligible if their abstract mentioned that data were collected during at least one year when the country received humanitarian funding through either the Consolidated Appeals Process (CAP) or Humanitarian Response Planning (HRP). If the year of data collection was unclear, abstracts were flagged but included in the full-text review $(n=54)$. Countries that received flash and regional appeals were only included if they also received either direct CAP or HRP funding. Systematic reviews and dissertations were not included, but their reference lists were reviewed to identify potentially relevant articles.

The 133 articles that met the criteria for full-text review were closely examined to determine if the article met exclusion criteria. First, the authors checked to see if each article was available in English. Articles were then re-reviewed to ensure data collection overlapped at some point during receipt of CAP and HRP funding between 2005 and 2020. Articles that did not make explicit note of when data were collected were excluded. However, articles were included if there was any data collection during a CAP or HRP year; thus, some articles may include data that were collected during CAP/HRP and nonCAP/HRP years. Similarly, included articles may include some degree of data collection before 2005 if simultaneous data collection coincided with a CAP/HRP year. Articles were also more rigorously examined based on if their definition of IPV aligned with the Inter-Agency Standing Committee (IASC) guidelines definition [1]. For example, articles needed to either include the explicit IPV terminology or describe how their measure of violence-particularly interpersonal and domestic violence-was restricted to intimate partners. Finally, analytical models that did not disaggregate by country were excluded.

\subsection{Data Extraction and Quality Assessment}

Each article that met inclusion and exclusion criteria was re-reviewed in full $(\mathrm{n}=51)$. Data extracted from each article related to the study's sampling framework, variable creation, and analysis methods. Using the Appraisal tool for Cross-Sectional Studies (AXIS tool) [36], all included articles were individually scored based on 20 quality assessment criteria.

\section{Findings}

\subsection{Overview of Included Articles}

The majority of included articles collected data in sub-Saharan Africa $(n=33)$. The remaining articles, including those with data from multiple regions, included data from Central and Southeast Asia $(n=7)$, the Middle East $(n=7)$, or the Caribbean $(n=5)$. It is worth noting that all articles from the Caribbean were from Haiti and no articles were published from Central nor South America. Further, while 48 countries received CAP or HRP funding between 2005 and 2020, only 18 had at least one article that met inclusion criteria. While articles that included data collection from 2020 to 2018 were limited, the years of data collection were roughly equal across the remaining years 2005-2017 with an average of six articles including data collected from each of these years.

All of the articles included women and/or girls within the sample and five also included men and/or boys. The vast majority of articles utilized cross-sectional data either from a cross-sectional $(72.55 \%, \mathrm{n}=37)$ or longitudinal study $(17.65 \%, \mathrm{n}=9)$. Only $9.80 \%$ $(\mathrm{n}=5)$ articles included analysis using longitudinal data. With $19.61 \%(\mathrm{n}=10)$ of articles using data from interventions, most of the remaining articles utilized population-based designs $(41.18 \%, \mathrm{n}=21)$ or non-population-based designs $(35.29 \%, \mathrm{n}=18)$. One article included data from an intervention study in Afghanistan and a non-population-based study design from Palestine. The final type of study design, a retrospective case review, was only used in one article that examined homicides in Maputo Province, Mozambique between 2016 and 2017.

The most common primary analysis model was multivariable logistic regression $(60.78 \%, \mathrm{n}=31)$. Ten articles $(19.61 \%)$ utilized other types of multivariable inferential modeling, including multivariable linear regression $(9.80 \%, n=5)$. One article included 
both multivariable linear and logistic regressions and another article utilized multivariable hierarchical regression. The primary models for the remaining nine articles that did not incorporate multivariable inferential modeling used either bivariate $(13.73 \%, \mathrm{n}=7)$ or descriptive $(3.92 \%, \mathrm{n}=2)$ analysis. See Table 1 for a comprehensive overview of the included articles.

Table 1. Overview of included studies.

\begin{tabular}{|c|c|c|c|c|c|c|c|c|c|}
\hline$\#$ & Citation & $\begin{array}{l}\text { Year(s) of } \\
\text { Data } \\
\text { Collection }\end{array}$ & $\begin{array}{c}\text { Country of } \\
\text { Data Collection }\end{array}$ & Study Type & Study Name & Temporality & $\begin{array}{l}\text { Primary Analysis } \\
\text { Model }\end{array}$ & Outcome Variable(s) ${ }^{++++}$ & $\begin{array}{c}\text { Quality } \\
\text { Assessment }\end{array}$ \\
\hline \multicolumn{10}{|c|}{ Middle East } \\
\hline 1 & $\begin{array}{c}\text { Goessmann, } \\
\text { Ibrahim, and } \\
\text { Neuner, 2020 } \\
\text { [37] }\end{array}$ & 2018-2019 & Iraqi Kurdistan & $\begin{array}{l}\text { Other non- } \\
\text { population } \\
\text { based design }\end{array}$ & Not stated & $\begin{array}{l}\text { Cross- } \\
\text { sectional }\end{array}$ & $\begin{array}{l}\text { Multi-variable } \\
\text { hierarchical regression }\end{array}$ & $\begin{array}{l}\text { Post-traumatic stress } \\
\text { disorder } \\
\text { Depression }\end{array}$ & $\begin{array}{l}\text { Top } \\
\text { category }\end{array}$ \\
\hline 2 & $\begin{array}{l}\text { Al-Atrushi } \\
\text { et al., } 2013 \text { [38] }\end{array}$ & 2009-2011 & Iraqi Kurdistan & $\begin{array}{l}\text { Other non- } \\
\text { population } \\
\text { based design }\end{array}$ & Not stated & $\begin{array}{l}\text { Cross- } \\
\text { sectional }\end{array}$ & $\begin{array}{l}\text { Descriptive analysis of } \\
\text { women who reported } \\
\text { lifetime IPV }\end{array}$ & $\begin{array}{ll}- & \text { Any physical injury } \\
- & \text { Cuts, bruises, or aches } \\
- & \text { Eye injuries, sprain, dislo- } \\
\text { cation, or burns } \\
\text { - Stab wounds, broken } \\
\text { bones, or broken teeth }\end{array}$ & $\begin{array}{l}\text { Lower- } \\
\text { middle } \\
\text { category }\end{array}$ \\
\hline 3 & $\begin{array}{l}\text { Zakar et al., } \\
2012[39]\end{array}$ & 2008-2009 & Pakistan & $\begin{array}{l}\text { Other non- } \\
\text { population } \\
\text { based design }\end{array}$ & Not stated & $\begin{array}{l}\text { Cross- } \\
\text { sectional }\end{array}$ & $\begin{array}{l}\text { Multi-variable logistic } \\
\text { regression (forms of } \\
\text { violence included } \\
\text { within separate } \\
\text { models) }\end{array}$ & $\begin{array}{l}\text { Past 6-mo poor } \\
\text { reproductive health } \\
\text { status }\end{array}$ & $\begin{array}{c}\text { Top } \\
\text { category }\end{array}$ \\
\hline 4 & $\begin{array}{l}\text { Zakar et al., } \\
2013 \text { [40] }\end{array}$ & 2008-2009 & Pakistan & $\begin{array}{l}\text { Other non- } \\
\text { population } \\
\text { based design }\end{array}$ & Not stated & $\begin{array}{l}\text { Cross- } \\
\text { sectional }\end{array}$ & $\begin{array}{l}\text { Multi-variable logistic } \\
\text { regression (different } \\
\text { forms of violence in } \\
\text { separate models) }\end{array}$ & Poor mental health & $\begin{array}{c}\text { Top } \\
\text { category }\end{array}$ \\
\hline 5 & $\begin{array}{l}\text { Sousa et al., } \\
2018 \text { [41] }\end{array}$ & 2007-2008 & Palestine & $\begin{array}{l}\text { Other non- } \\
\text { population } \\
\text { based design }\end{array}$ & Not stated & $\begin{array}{l}\text { Cross- } \\
\text { sectional }\end{array}$ & $\begin{array}{l}\text { Multi-variable } \\
\text { regression (all forms of } \\
\text { violence included } \\
\text { within same model) }\end{array}$ & $\begin{array}{l}\text { Mental Component } \\
\text { Summary (interval) } \\
\text { Distress (interval) } \\
\text { PTSD (interval) }\end{array}$ & $\begin{array}{l}\text { Lower- } \\
\text { middle } \\
\text { category }\end{array}$ \\
\hline 6 & $\begin{array}{l}\text { Falb et al., } 2019 \\
{[42]}\end{array}$ & 2018 & Syria & $\begin{array}{l}\text { Intervention } \\
\text { study }\end{array}$ & $\begin{array}{l}\text { Not stated } \\
\text { (cash transfer } \\
\text { program) }\end{array}$ & $\begin{array}{l}\text { Cross- } \\
\text { sectional } \\
\text { (baseline) }\end{array}$ & $\begin{array}{l}\text { Multi-variable linear } \\
\text { regression (all forms of } \\
\text { violence included } \\
\text { within same model) }\end{array}$ & $\begin{array}{l}\text { Past 2-week depression } \\
\text { symptoms (ratio) }\end{array}$ & $\begin{array}{c}\text { Top } \\
\text { category }\end{array}$ \\
\hline \multicolumn{10}{|c|}{ West Africa and the Sahel Region } \\
\hline 7 & $\begin{array}{l}\text { Fiorentino } \\
\text { et al., } 2019 \text { [43] }\end{array}$ & 2014 & Cameroon & $\begin{array}{l}\text { Other non- } \\
\text { population } \\
\text { based design }\end{array}$ & $\begin{array}{l}\text { ANRS-12288 } \\
\text { EVOLCam }\end{array}$ & $\begin{array}{l}\text { Cross- } \\
\text { sectional }\end{array}$ & $\begin{array}{l}\text { Multi-variable logistic } \\
\text { regression }\end{array}$ & Recent ATI $\geq 1$-month & $\begin{array}{c}\text { Top } \\
\text { category }\end{array}$ \\
\hline 8 & $\begin{array}{c}\text { Peltzer and } \\
\text { Pengpid, } 2014 \\
\text { [44] }\end{array}$ & 2011-2012 & Ivory Coast & $\begin{array}{l}\text { Population- } \\
\text { based } \\
\text { design }\end{array}$ & $\begin{array}{l}\text { Demographic } \\
\text { Health } \\
\text { Survey }\end{array}$ & $\begin{array}{l}\text { Cross- } \\
\text { sectional }\end{array}$ & $\begin{array}{l}\text { Multi-variable logistic } \\
\text { regression }\end{array}$ & $\begin{array}{ll}- & \text { Lifetime any IPV } \\
- & \text { Lifetime physical IPV } \\
- & \text { Lifetime sexual IPV } \\
- & \text { Lifetime emotional IPV } \\
- & \text { Lifetime one type of IPV } \\
- & \text { Lifetime two or three } \\
& \text { types of IPV }\end{array}$ & $\begin{array}{l}\text { Upper- } \\
\text { middle } \\
\text { category }\end{array}$ \\
\hline 9 & $\begin{array}{l}\text { Alenko, et al., } \\
2020[45]\end{array}$ & 2019 & Ethiopia & $\begin{array}{l}\text { Other non- } \\
\text { population } \\
\text { based design }\end{array}$ & Not stated & $\begin{array}{l}\text { Cross- } \\
\text { sectional }\end{array}$ & $\begin{array}{l}\text { Multi-variable logistic } \\
\text { regression }\end{array}$ & $\begin{array}{l}\text { Child emotional and } \\
\text { behavioral problems }\end{array}$ & $\begin{array}{l}\text { Top } \\
\text { category }\end{array}$ \\
\hline 10 & $\begin{array}{l}\text { Gebreslasie } \\
\text { et al., } 2020[46]\end{array}$ & $2017-2018$ & Ethiopia & $\begin{array}{l}\text { Other non- } \\
\text { population } \\
\text { based design }\end{array}$ & Not stated & $\begin{array}{l}\text { Cross- } \\
\text { sectional }\end{array}$ & $\begin{array}{l}\text { Multi-variable logistic } \\
\text { regression }\end{array}$ & Stillbirth & $\begin{array}{l}\text { Top } \\
\text { category }\end{array}$ \\
\hline 11 & $\begin{array}{l}\text { Sipsma et al., } \\
2013 \text { [47] }\end{array}$ & 2007 & Liberia & $\begin{array}{l}\text { Population- } \\
\text { based } \\
\text { design }\end{array}$ & $\begin{array}{l}\text { Demographic } \\
\text { Health } \\
\text { Survey }\end{array}$ & $\begin{array}{l}\text { Cross- } \\
\text { sectional }\end{array}$ & $\begin{array}{l}\text { Multi-variable logistic } \\
\text { regression (all forms of } \\
\text { violence included } \\
\text { within same model) }\end{array}$ & $\begin{array}{l}\text { Past } 12 \text {-mo visit to health } \\
\text { facility for self or own } \\
\text { children }\end{array}$ & $\begin{array}{l}\text { Upper- } \\
\text { middle } \\
\text { category }\end{array}$ \\
\hline 12 & $\begin{array}{c}\text { Sobkoviak, } \\
\text { Yount, and } \\
\text { Halim, } 2012 \\
{[48]}\end{array}$ & 2007 & Liberia & $\begin{array}{l}\text { Population- } \\
\text { based } \\
\text { design }\end{array}$ & $\begin{array}{l}\text { Demographic } \\
\text { Health } \\
\text { Survey }\end{array}$ & $\begin{array}{l}\text { Cross- } \\
\text { sectional }\end{array}$ & $\begin{array}{l}\text { Multi-variable linear or } \\
\text { logistic regression (all } \\
\text { forms of violence } \\
\text { included within same } \\
\text { model) }\end{array}$ & $\begin{array}{ll}\text { - } & \text { Child's weight-for-height } \\
\text { (interval) } \\
\text { Child's weight-for-age } \\
\text { (interval) } \\
\text { Child's height-for-age } \\
\text { (interval) } \\
\text { Child's z-scores were less } \\
\text { than two SDs below the } \\
\text { mean for wasted } \\
\text { Child's z-scores were less } \\
\text { than two SDs below the } \\
\text { mean for underweight } \\
\text { Child's z-scores were less } \\
\text { than two SDs below the } \\
\text { mean for stunted }\end{array}$ & $\begin{array}{l}\text { Upper- } \\
\text { middle } \\
\text { category }\end{array}$ \\
\hline 13 & $\begin{array}{l}\text { Hayes and van } \\
\text { Baak, } 2017 \text { [49] }\end{array}$ & 2012-2013 & Mali & $\begin{array}{l}\text { Population- } \\
\text { based } \\
\text { design }\end{array}$ & $\begin{array}{l}\text { Demographic } \\
\text { Health } \\
\text { Survey }\end{array}$ & $\begin{array}{l}\text { Cross- } \\
\text { sectional }\end{array}$ & Logistic regression & $\begin{array}{l}\text { Lifetime sexual IPV } \\
\text { Lifetime physical IPV }\end{array}$ & $\begin{array}{l}\text { Upper- } \\
\text { middle } \\
\text { category }\end{array}$ \\
\hline 14 & $\begin{array}{l}\text { DeLong et al., } \\
2020 \text { [50] }\end{array}$ & 2016 & Niger & $\begin{array}{l}\text { Other non- } \\
\text { population- } \\
\text { based } \\
\text { design }\end{array}$ & RMA study & $\begin{array}{l}\text { Cross- } \\
\text { sectional } \\
\text { (baseline) }\end{array}$ & $\begin{array}{l}\text { Multi-variable logistic } \\
\text { regression (violence } \\
\text { typologies included } \\
\text { within same model \& } \\
\text { separate models for } \\
\text { girls 15-16 \& 17-19 } \\
\text { years old) }\end{array}$ & $\begin{array}{ll}\text { - } & \text { Current use of modern } \\
\text { family planning } \\
\text { - } & \text { Lifetime UIP experience }\end{array}$ & $\begin{array}{l}\text { Upper- } \\
\text { middle } \\
\text { category }\end{array}$ \\
\hline
\end{tabular}


Table 1. Cont.

\begin{tabular}{|c|c|c|c|c|c|c|c|c|c|c|}
\hline$\#$ & Citation & $\begin{array}{l}\text { Year(s) of } \\
\text { Data } \\
\text { Collection }\end{array}$ & $\begin{array}{c}\text { Country of } \\
\text { Data Collection }\end{array}$ & Study Type & Study Name & Temporality & $\begin{array}{l}\text { Primary Analysis } \\
\text { Model }\end{array}$ & & Outcome Variable(s) ${ }^{++++}$ & $\begin{array}{c}\text { Quality } \\
\text { Assessment }\end{array}$ \\
\hline 15 & $\begin{array}{l}\text { Silverman } \\
\text { et al., } 2020 \text { [51] }\end{array}$ & 2016 & Niger & $\begin{array}{l}\text { Intervention } \\
\text { study }\end{array}$ & RMA study & $\begin{array}{c}\text { Cross- } \\
\text { sectional } \\
\text { (baseline) }\end{array}$ & $\begin{array}{l}\text { Multi-variable logistic } \\
\text { regression (violence } \\
\text { typologies included } \\
\text { within same model) }\end{array}$ & - & $\begin{array}{l}\text { Current use of reversible } \\
\text { modern family planning } \\
\text { method }\end{array}$ & $\begin{array}{l}\text { Upper- } \\
\text { middle } \\
\text { category }\end{array}$ \\
\hline 16 & $\begin{array}{l}\text { Kana, et al., } \\
2020 \text { [52] }\end{array}$ & 2017-2019 & Nigeria & $\begin{array}{c}\text { Other non- } \\
\text { population } \\
\text { based design }\end{array}$ & Not stated & $\begin{array}{l}\text { Cross- } \\
\text { sectional }\end{array}$ & $\begin{array}{l}\text { Multi-variable logistic } \\
\text { regression }\end{array}$ & - & $\begin{array}{l}\text { Birth weight among term } \\
\text { births }\end{array}$ & $\begin{array}{c}\text { Top } \\
\text { category }\end{array}$ \\
\hline 17 & $\begin{array}{l}\text { Abiodun et al., } \\
2020[53]\end{array}$ & 2019 & Nigeria & $\begin{array}{l}\text { Other non- } \\
\text { population } \\
\text { based design }\end{array}$ & not stated & $\begin{array}{l}\text { Cross- } \\
\text { sectional }\end{array}$ & $\begin{array}{l}\text { Multi-variable logistic } \\
\text { regression }\end{array}$ & - & $\begin{array}{l}\text { Past 12-mo experience of } \\
\text { IPV }\end{array}$ & $\begin{array}{l}\text { Top } \\
\text { category }\end{array}$ \\
\hline \multicolumn{11}{|c|}{ Southern and Eastern Africa } \\
\hline 18 & $\begin{array}{c}\text { Kidman, } \\
\text { Palermo and } \\
\text { Bertrand, } 2015 \\
{[54]}\end{array}$ & 2007 & $\begin{array}{l}\text { Democratic } \\
\text { Republic of } \\
\text { Congo }\end{array}$ & $\begin{array}{l}\text { Population- } \\
\text { based } \\
\text { design }\end{array}$ & $\begin{array}{l}\text { Demographic } \\
\text { Health } \\
\text { Survey }\end{array}$ & $\begin{array}{l}\text { Cross- } \\
\text { sectional }\end{array}$ & $\begin{array}{l}\text { Multi-variable logistic } \\
\text { regression (separate } \\
\text { models for each form } \\
\text { of IPV) }\end{array}$ & - & $\begin{array}{l}\text { Current use of modern } \\
\text { contraception }\end{array}$ & $\begin{array}{l}\text { Upper- } \\
\text { middle } \\
\text { category }\end{array}$ \\
\hline 19 & $\begin{array}{l}\text { Fleming et al., } \\
2015 \text { [55] }\end{array}$ & 2009-2012 & $\begin{array}{l}\text { Democratic } \\
\text { Republic of } \\
\text { Congo }^{+}\end{array}$ & $\begin{array}{l}\text { Intervention } \\
\text { study }\end{array}$ & IMAGES & $\begin{array}{c}\text { Cross- } \\
\text { sectional } \\
\text { (baseline) }\end{array}$ & $\begin{array}{l}\text { Multi-variable logistic } \\
\text { regression }\end{array}$ & - & $\begin{array}{l}\text { Lifetime physical IPV } \\
\text { perpetration }\end{array}$ & $\begin{array}{l}\text { Top } \\
\text { category }\end{array}$ \\
\hline 20 & $\begin{array}{l}\text { Johnson et al., } \\
2010[56]\end{array}$ & 2010 & $\begin{array}{l}\text { Democratic } \\
\text { Republic of } \\
\text { Congo }\end{array}$ & $\begin{array}{c}\text { Population- } \\
\text { based design } \\
++\end{array}$ & Not stated & $\begin{array}{l}\text { Cross- } \\
\text { sectional }\end{array}$ & $\begin{array}{l}\text { Bivariate comparisons } \\
\text { of prevalence } \\
\text { calculated using the } \\
\text { adjusted Wald test of } \\
\text { association (modeled } \\
\text { for males and females) }\end{array}$ & $\begin{array}{l}- \\
-\end{array}$ & $\begin{array}{l}\text { Substance abuse } \\
\text { Major depressive } \\
\text { disorder } \\
\text { PTSD } \\
\text { Suicidal ideation } \\
\text { Suicide attempt } \\
\end{array}$ & $\begin{array}{l}\text { Upper- } \\
\text { middle } \\
\text { category }\end{array}$ \\
\hline 21 & $\begin{array}{l}\text { Gichane et al., } \\
2018 \text { [57] }\end{array}$ & 2013-2014 & $\begin{array}{l}\text { Democratic } \\
\text { Republic of } \\
\text { Congo }\end{array}$ & $\begin{array}{l}\text { Intervention } \\
\text { study }\end{array}$ & $\begin{array}{l}\text { Unnamed- } \\
\text { Conditional } \\
\text { Cash Transfer } \\
\text { study }\end{array}$ & Longitudinal & $\begin{array}{l}\text { Adjusted prevalence } \\
\text { ratios (forms of } \\
\text { violence included } \\
\text { within separate } \\
\text { models) }\end{array}$ & - & $\begin{array}{l}\text { Viral suppression at } \\
\text { 6-week follow-up } \\
\text { Uptake of PMTCT } \\
\text { services at 6-week follow } \\
\text { up } \\
\text { ARV adherence measured } \\
\text { at 6-week follow up }\end{array}$ & $\begin{array}{c}\text { Top } \\
\text { category }\end{array}$ \\
\hline 22 & $\begin{array}{l}\text { Tiruneh et al., } \\
2018 \text { [58] }\end{array}$ & 2013-2014 & $\begin{array}{l}\text { Democratic } \\
\text { Republic of the } \\
\text { Congo }\end{array}$ & $\begin{array}{l}\text { Population- } \\
\text { based } \\
\text { design }\end{array}$ & $\begin{array}{l}\text { Demographic } \\
\text { Health } \\
\text { Survey }\end{array}$ & $\begin{array}{l}\text { Cross- } \\
\text { sectional }\end{array}$ & $\begin{array}{l}\text { Multi-variable logistic } \\
\text { regression (two } \\
\text { models: one with } \\
\text { physical, sexual, and } \\
\text { emotional IPV as } \\
\text { separate variables, and } \\
\text { the second with any } \\
\text { IPV) }\end{array}$ & $\begin{array}{l}- \\
- \\
- \\
-\end{array}$ & $\begin{array}{l}\text { Lifetime unintended } \\
\text { pregnancy } \\
\text { Lifetime pregnancy loss } \\
\text { Lifetime physical IPV } \\
\text { Lifetime sexual IPV } \\
\text { Lifetime emotional IPV } \\
\text { Lifetime any IPV }\end{array}$ & $\begin{array}{l}\text { Upper- } \\
\text { middle } \\
\text { category }\end{array}$ \\
\hline 23 & $\begin{array}{l}\text { Mpody et al., } \\
2019 \text { [59] }\end{array}$ & 2016-2018 & $\begin{array}{l}\text { Democratic } \\
\text { Republic of } \\
\text { Congo }\end{array}$ & $\begin{array}{l}\text { Intervention } \\
\text { study }\end{array}$ & $\begin{array}{l}\text { CQI-PMTCT } \\
\text { study }\end{array}$ & $\begin{array}{l}\text { Cross- } \\
\text { sectional } \\
\text { (baseline) }\end{array}$ & $\begin{array}{l}\text { Multi-variable logistic } \\
\text { regression (separate } \\
\text { models based on } \\
\text { pregnancy status } \\
\text { during enrollment) }\end{array}$ & - & $\begin{array}{l}\text { Current presence of } \\
\text { HBsAG in blood }\end{array}$ & $\begin{array}{l}\text { Upper- } \\
\text { middle } \\
\text { category }\end{array}$ \\
\hline 24 & $\begin{array}{l}\text { Onsomu et al., } \\
2015 \text { [60] }\end{array}$ & 2008-2009 & Kenya & $\begin{array}{l}\text { Population- } \\
\text { based } \\
\text { design }\end{array}$ & $\begin{array}{l}\text { Demographic } \\
\text { Health } \\
\text { Survey }\end{array}$ & $\begin{array}{l}\text { Cross- } \\
\text { sectional }\end{array}$ & $\begin{array}{l}\text { Multi-variable logistic } \\
\text { regression (separate } \\
\text { models for each form } \\
\text { of IPV) }\end{array}$ & - & HIV serostatus & $\begin{array}{l}\text { Top } \\
\text { category }\end{array}$ \\
\hline 25 & $\begin{array}{l}\text { Shi, } \\
\text { Kouyoumdjian } \\
\text { and Dushoff, } \\
2013 \text { [61] }\end{array}$ & 2008-2009 & Kenya & $\begin{array}{l}\text { Population- } \\
\text { based } \\
\text { design }\end{array}$ & $\begin{array}{l}\text { Demographic } \\
\text { Health } \\
\text { Survey }\end{array}$ & $\begin{array}{l}\text { Cross- } \\
\text { sectional }\end{array}$ & $\begin{array}{l}\text { Generalized linear } \\
\text { mixed models }\end{array}$ & - & Current $\mathrm{HIV}^{+}$status & $\begin{array}{l}\text { Top } \\
\text { category }\end{array}$ \\
\hline 26 & $\begin{array}{l}\text { Seff and Stark, } \\
2019 \text { [62] }\end{array}$ & 2010 & Kenya $^{+}$ & $\begin{array}{l}\text { Population- } \\
\text { based design } \\
++\end{array}$ & $\begin{array}{l}\text { Violence } \\
\text { Against } \\
\text { Children } \\
\text { Surveys }\end{array}$ & $\begin{array}{l}\text { Cross- } \\
\text { sectional }\end{array}$ & $\begin{array}{l}\text { Multi-variable logistic } \\
\text { regression (separate } \\
\text { models for males and } \\
\text { females) }\end{array}$ & - & Lifetime suicide ideation & $\begin{array}{l}\text { Upper- } \\
\text { middle } \\
\text { category }\end{array}$ \\
\hline 27 & $\begin{array}{l}\text { Phillips- } \\
\text { Howard et al., } \\
2015 \text { [63] }\end{array}$ & 2011-2012 & Kenya & $\begin{array}{c}\text { Other non- } \\
\text { population } \\
\text { based design }\end{array}$ & Not stated & $\begin{array}{l}\text { Cross- } \\
\text { sectional }\end{array}$ & $\begin{array}{l}\text { Multi-variable logistic } \\
\text { regression (separate } \\
\text { models for married } \\
\text { and never-married } \\
\text { women) }\end{array}$ & $\begin{array}{l}- \\
-\end{array}$ & $\begin{array}{l}\text { Commercial pad use } \\
\text { Commercial pad } \\
\text { provided by sexual } \\
\text { partner }\end{array}$ & $\begin{array}{l}\text { Upper- } \\
\text { middle } \\
\text { category }\end{array}$ \\
\hline 28 & $\begin{array}{l}\text { Kinuthia et al., } \\
2018 \text { [64] }\end{array}$ & 2013 & Kenya & $\begin{array}{c}\text { Other non- } \\
\text { population } \\
\text { based design }\end{array}$ & Not stated & $\begin{array}{l}\text { Cross- } \\
\text { sectional }\end{array}$ & $\begin{array}{l}\text { Multi-variable logistic } \\
\text { regression }\end{array}$ & - & $\begin{array}{l}\text { Current non-disclosure of } \\
\text { HIV- status }\end{array}$ & $\begin{array}{l}\text { Top } \\
\text { category }\end{array}$ \\
\hline 29 & $\begin{array}{l}\text { Goyette et al., } \\
2018 \text { [65] }\end{array}$ & 2013-2015 & Kenya & $\begin{array}{l}\text { Intervention } \\
\text { study }\end{array}$ & not stated & Longitudinal & $\begin{array}{c}\text { Log-binomial } \\
\text { generalized estimated } \\
\text { equation }\end{array}$ & - & $\begin{array}{l}\text { Partner tested for HIV } \\
\text { Partner newly diagnosed } \\
\text { with HIV } \\
\text { Partner new linkage to } \\
\text { care for HIV }\end{array}$ & $\begin{array}{l}\text { Upper- } \\
\text { middle } \\
\text { category }\end{array}$ \\
\hline 30 & $\begin{array}{l}\text { Wagman et al., } \\
2016[66]\end{array}$ & 1999-2006 & Uganda & $\begin{array}{l}\text { Intervention } \\
\text { study }\end{array}$ & $\begin{array}{l}\text { RCCS in } \\
\text { Rakai }\end{array}$ & Longitudinal & $\begin{array}{l}\text { Multi-variable linear } \\
\text { regressions (all forms } \\
\text { of violence included } \\
\text { within same model } \\
\text { and model includes an } \\
\text { aggregate of each wave } \\
\text { of data collection) } \\
\end{array}$ & - & $\begin{array}{l}\text { Divorce or separation } \\
\text { from husband or primary } \\
\text { sexual partner }\end{array}$ & $\begin{array}{l}\text { Upper- } \\
\text { middle } \\
\text { category }\end{array}$ \\
\hline 31 & $\begin{array}{l}\text { Kouyoumdjian } \\
\text { et al., } 2013 \text { [67] }\end{array}$ & 2000-2009 & Uganda & $\begin{array}{l}\text { Intervention } \\
\text { study }\end{array}$ & $\begin{array}{l}\text { RCCS in } \\
\text { Rakai }\end{array}$ & Longitudinal & $\begin{array}{c}\text { Multi-variable } \\
\text { population attributable } \\
\text { fraction (separate } \\
\text { models for forms of } \\
\text { IPV) }\end{array}$ & 5 & $\begin{array}{l}\text { Past-year HIV infection } \\
\text { Lifetime HIV infection }\end{array}$ & $\begin{array}{c}\text { Top } \\
\text { category }\end{array}$ \\
\hline
\end{tabular}


Table 1. Cont.

\begin{tabular}{|c|c|c|c|c|c|c|c|c|c|c|}
\hline$\#$ & Citation & $\begin{array}{l}\text { Year(s) of } \\
\text { Data } \\
\text { Collection }\end{array}$ & $\begin{array}{c}\text { Country of } \\
\text { Data Collection }\end{array}$ & Study Type & Study Name & Temporality & $\begin{array}{l}\text { Primary Analysis } \\
\text { Model }\end{array}$ & & Outcome Variable(s) ${ }^{++++}$ & $\begin{array}{l}\text { Quality } \\
\text { Assessment }\end{array}$ \\
\hline 32 & $\begin{array}{c}\text { Speizer, } 2010 \\
{[68]}\end{array}$ & 2006 & Uganda & $\begin{array}{c}\text { Population- } \\
\text { based design } \\
++\end{array}$ & $\begin{array}{l}\text { Demographic } \\
\text { Health } \\
\text { Survey }\end{array}$ & $\begin{array}{l}\text { Cross- } \\
\text { sectional }\end{array}$ & $\begin{array}{l}\text { Multi-variable } \\
\text { multinomial logistic } \\
\text { regression (separate } \\
\text { models for married or } \\
\text { unionized men and } \\
\text { women) }\end{array}$ & - & $\begin{array}{l}\text { Attitudinal acceptance of } \\
\text { violence }\end{array}$ & $\begin{array}{l}\text { Upper- } \\
\text { middle } \\
\text { category }\end{array}$ \\
\hline 33 & $\begin{array}{c}\text { Macucha and } \\
\text { Taunde, 2020 } \\
{[69]}\end{array}$ & 2016-2017 & Mozambique & $\begin{array}{l}\text { Retrospective } \\
\text { case review }\end{array}$ & Not stated & $\begin{array}{l}\text { Cross- } \\
\text { sectional }\end{array}$ & $\begin{array}{l}\text { Descriptive analysis of } \\
\text { autopsy reports }\end{array}$ & - & Homicide victimization & $\begin{array}{l}\text { Upper- } \\
\text { middle } \\
\text { category }\end{array}$ \\
\hline 34 & $\begin{array}{c}\text { Adjiwan and } \\
\text { N'Bouke, } 2015 \\
\text { [70] }\end{array}$ & 2010 & Zimbabwe $^{+}$ & $\begin{array}{l}\text { Population- } \\
\text { based } \\
\text { design }\end{array}$ & $\begin{array}{l}\text { Demographic } \\
\text { Health } \\
\text { Survey }\end{array}$ & $\begin{array}{l}\text { Cross- } \\
\text { sectional }\end{array}$ & $\begin{array}{l}\text { Multi-variable probit } \\
\text { model }\end{array}$ & - & $\begin{array}{l}\text { Current use of modern, } \\
\text { non-permanent } \\
\text { contraception }\end{array}$ & $\begin{array}{l}\text { Upper- } \\
\text { middle } \\
\text { category }\end{array}$ \\
\hline 35 & $\begin{array}{l}\text { Shamu et al., } \\
2016 \text { [71] }\end{array}$ & 2011 & Zimbabwe & $\begin{array}{c}\text { Other non- } \\
\text { population } \\
\text { based design }\end{array}$ & not stated & $\begin{array}{l}\text { Cross- } \\
\text { sectional }\end{array}$ & $\begin{array}{l}\text { Multi-variable logistic } \\
\text { regression (all forms of } \\
\text { violence included } \\
\text { within same model) }\end{array}$ & - & $\begin{array}{l}\text { Past 4-week depression } \\
\text { Lifetime suicidal } \\
\text { thoughts }\end{array}$ & $\begin{array}{l}\text { Upper- } \\
\text { middle } \\
\text { category }\end{array}$ \\
\hline 36 & $\begin{array}{l}\text { Shamu et al., } \\
2018 \text { [72] }\end{array}$ & 2011 & Zimbabwe & $\begin{array}{l}\text { Other non- } \\
\text { population } \\
\text { based design }\end{array}$ & Not stated & $\begin{array}{l}\text { Cross- } \\
\text { sectional }\end{array}$ & $\begin{array}{l}\text { Multi-variable logistic } \\
\text { regression (all forms of } \\
\text { violence included } \\
\text { within same model) }\end{array}$ & $\begin{array}{l}- \\
- \\
- \\
-\end{array}$ & $\begin{array}{l}\text { Lifetime history of } \\
\text { miscarriage and/or } \\
\text { stillbirth } \\
\text { Lifetime history of a } \\
\text { miscarriage } \\
\text { Lifetime history of } \\
\text { neonatal death } \\
\text { Lifetime history of a } \\
\text { neonatal death, } \\
\text { miscarriage, and/or } \\
\text { stillbirth } \\
\text { History of an unplanned } \\
\text { pregnancy } \\
\text { History of late booking } \\
\text { into care } \\
\text { History of never booking } \\
\text { into care } \\
\text { Recent negative birth } \\
\text { outcome } \\
\text { Recent low birth weight }\end{array}$ & $\begin{array}{l}\text { Upper- } \\
\text { middle } \\
\text { category }\end{array}$ \\
\hline 37 & $\begin{array}{l}\text { Nyamukoho } \\
\text { et al., } 2019 \text { [73] }\end{array}$ & 2016 & Zimbabwe & $\begin{array}{l}\text { Other non- } \\
\text { population } \\
\text { based design }\end{array}$ & Not stated & $\begin{array}{l}\text { Cross } \\
\text { sectional }\end{array}$ & Logistic regression & - & Depression & $\begin{array}{l}\text { Lower- } \\
\text { middle } \\
\text { category }\end{array}$ \\
\hline & & & & & Caribbean & & & & & \\
\hline 38 & $\begin{array}{l}\text { Kayibanda and } \\
\text { Alary, } 2020[74]\end{array}$ & $\begin{array}{l}\text { 2000, } 2005 \\
\quad 2012\end{array}$ & Haiti & $\begin{array}{l}\text { Population- } \\
\text { based } \\
\text { design }\end{array}$ & $\begin{array}{l}\text { Demographic } \\
\text { Health } \\
\text { Survey }\end{array}$ & $\begin{array}{l}\text { Cross- } \\
\text { sectional }\end{array}$ & $\begin{array}{l}\text { Multi-variable analysis } \\
\text { adjusted prevalence } \\
\text { ratios (all forms of } \\
\text { violence included } \\
\text { within same model) }\end{array}$ & - & $\begin{array}{l}\text { Lifetime physical IPV } \\
\text { perpetration }\end{array}$ & $\begin{array}{l}\text { Upper- } \\
\text { middle } \\
\text { category }\end{array}$ \\
\hline 39 & Gage, 2016 [75] & 2013 & Haiti & $\begin{array}{l}\text { Intervention } \\
\text { study }{ }^{++}\end{array}$ & SAFE Dates & $\begin{array}{l}\text { Cross- } \\
\text { sectional } \\
\text { (baseline) }\end{array}$ & $\begin{array}{l}\text { Multi-variable logistic } \\
\text { regression (both forms } \\
\text { of violence included in } \\
\text { model and separate } \\
\text { models for males and } \\
\text { females) }\end{array}$ & - & $\begin{array}{l}\text { Lifetime psychological } \\
\text { IPV perpetration (ratio) } \\
\text { Lifetime physical/sexual } \\
\text { IPV perpetration (ratio) }\end{array}$ & $\begin{array}{l}\text { Upper- } \\
\text { middle } \\
\text { category }\end{array}$ \\
\hline 40 & $\begin{array}{l}\text { Saxena et al., } \\
2019 \text { [76] }\end{array}$ & 2013 & Haiti & $\begin{array}{l}\text { Intervention } \\
\text { study }\end{array}$ & GHESKIO & $\begin{array}{l}\text { Cross- } \\
\text { sectional } \\
\text { (baseline) }\end{array}$ & $\begin{array}{c}\text { Multi-variable logistic } \\
\text { regression (all violence } \\
\text { forms included within } \\
\text { same model) }\end{array}$ & - & Current $\mathrm{HIV}^{+}$status & $\begin{array}{l}\text { Upper- } \\
\text { middle } \\
\text { category }\end{array}$ \\
\hline 41 & $\begin{array}{l}\text { Occean et al., } \\
2020[77]\end{array}$ & 2016-2017 & Haiti & $\begin{array}{l}\text { Population- } \\
\text { based } \\
\text { design }\end{array}$ & $\begin{array}{l}\text { Demographic } \\
\text { Health } \\
\text { Survey }\end{array}$ & $\begin{array}{l}\text { Cross- } \\
\text { sectional }\end{array}$ & $\begin{array}{l}\text { Multi-variable logistic } \\
\text { regression }\end{array}$ & - & Lifetime any IPV & $\begin{array}{l}\text { Upper- } \\
\text { middle } \\
\text { category }\end{array}$ \\
\hline 42 & $\begin{array}{l}\text { Zalla et al., } \\
2019 \text { [78] }\end{array}$ & $2016-2017$ & Haiti & $\begin{array}{l}\text { Other non- } \\
\text { population } \\
\text { based design }\end{array}$ & $\begin{array}{l}\text { Local AIDS } \\
\text { Control } \\
\text { Efforts } \\
\text { (PLACE) } \\
\text { study }\end{array}$ & $\begin{array}{l}\text { Cross- } \\
\text { sectional }\end{array}$ & $\begin{array}{l}\text { Bivariate log-binomial } \\
\text { regression (separate } \\
\text { models for each } \\
\text { group-MSM, FSW, } \\
\text { TWG) }\end{array}$ & - & $\begin{array}{l}\text { Viral load from dried } \\
\text { blood spots (ratio) }\end{array}$ & $\begin{array}{l}\text { Upper- } \\
\text { middle } \\
\text { category }\end{array}$ \\
\hline \multicolumn{11}{|c|}{ Central and Southeast Asia } \\
\hline 43 & $\begin{array}{l}\text { Bellizzi et al., } \\
2019 \text { [79] }\end{array}$ & 2015 & Afghanistan $^{+}$ & $\begin{array}{l}\text { Population- } \\
\text { based } \\
\text { design }\end{array}$ & $\begin{array}{l}\text { Demographic } \\
\text { Health } \\
\text { Survey }\end{array}$ & $\begin{array}{l}\text { Cross- } \\
\text { sectional }\end{array}$ & $\begin{array}{l}\text { Multi-variable logistic } \\
\text { regression }\end{array}$ & - & $\begin{array}{l}\text { Past 3-year eclampsia } \\
\text { around childbirth }\end{array}$ & $\begin{array}{l}\text { Upper- } \\
\text { middle } \\
\text { category }\end{array}$ \\
\hline 44 & $\begin{array}{l}\text { Gibbs, Corboz, } \\
\text { and Jewkes, } \\
2018 \text { [80] }\end{array}$ & 2016-2017 & Afghanistan & $\begin{array}{l}\text { Intervention } \\
\text { study }\end{array}$ & WfWI trial & $\begin{array}{l}\text { Cross- } \\
\text { sectional } \\
\text { (baseline) }\end{array}$ & $\begin{array}{l}\text { Multi-variable linear } \\
\text { regressions (separate } \\
\text { models for forms and } \\
\text { rates of IPV) }\end{array}$ & $\begin{array}{l}- \\
- \\
-\end{array}$ & $\begin{array}{l}\text { Past-week depression } \\
\text { Past-week PTSD } \\
\text { symptoms } \\
\text { Current life satisfaction } \\
\text { (ratio) } \\
\text { Lifetime suicidal ideation } \\
\text { Current overall health } \\
\text { Current functional } \\
\text { limitations or disability } \\
\text { (ratio) }\end{array}$ & $\begin{array}{l}\text { Upper- } \\
\text { middle } \\
\text { category }\end{array}$ \\
\hline 45 & $\begin{array}{l}\text { Aye, et al., } \\
2020[81]\end{array}$ & 2016 & Myanmar & $\begin{array}{c}\text { Population- } \\
\text { based design } \\
++\end{array}$ & $\begin{array}{l}\text { Demographic } \\
\text { Health } \\
\text { Survey }\end{array}$ & $\begin{array}{l}\text { Cross- } \\
\text { sectional }\end{array}$ & $\begin{array}{l}\text { Multi-variablelinear } \\
\text { regression }\end{array}$ & - & Mental distress & $\begin{array}{l}\text { Top } \\
\text { category }\end{array}$ \\
\hline 46 & $\begin{array}{l}\text { Tsai, Cappa } \\
\text { and Petrowski, } \\
2016 \text { [82] }\end{array}$ & 2013 & Philippines & $\begin{array}{l}\text { Population- } \\
\text { based } \\
\text { design }\end{array}$ & $\begin{array}{c}\text { Demographic } \\
\text { Health } \\
\text { Survey }\end{array}$ & $\begin{array}{l}\text { Cross- } \\
\text { sectional }\end{array}$ & $\begin{array}{l}\text { Multi-variable logistic } \\
\text { regression (separate } \\
\text { models for each form } \\
\text { of IPV) }\end{array}$ & - & $\begin{array}{l}\text { Current use of } \\
\text { contraception }\end{array}$ & $\begin{array}{l}\text { Upper- } \\
\text { middle } \\
\text { category }\end{array}$ \\
\hline
\end{tabular}


Table 1. Cont.

\begin{tabular}{|c|c|c|c|c|c|c|c|c|c|}
\hline$\#$ & Citation & $\begin{array}{l}\text { Year(s) of } \\
\text { Data } \\
\text { Collection }\end{array}$ & $\begin{array}{l}\text { Country of Data } \\
\text { Collection }\end{array}$ & Study Type & Study Name & Temporality & $\begin{array}{c}\text { Primary Analysis } \\
\text { Model }\end{array}$ & Outcome Variable(s) $^{++++}$ & $\begin{array}{l}\text { Quality } \\
\text { Assess- } \\
\text { ment }\end{array}$ \\
\hline 47 & $\begin{array}{c}\text { Falb et al., } 2014 \\
{[83]}\end{array}$ & 2008 & $\begin{array}{c}\text { Thai-Myanmar } \\
\text { Border }\end{array}$ & $\begin{array}{c}\text { Other non- } \\
\text { population } \\
\text { based design }\end{array}$ & $\begin{array}{l}\text { Reproductive } \\
\text { Health } \\
\text { Assessment } \\
\text { Toolkit for } \\
\text { Conflict- } \\
\text { Affected } \\
\text { women }\end{array}$ & $\begin{array}{l}\text { Cross- } \\
\text { sectional }\end{array}$ & $\begin{array}{c}\text { Multi-variable } \\
\text { generalized estimated } \\
\text { equations }\end{array}$ & $\begin{array}{l}\text { Symptoms of pregnancy } \\
\text { complications for the } \\
\text { most recent pregnancy } \\
\text { that resulted in a live } \\
\text { birth in the last two years }\end{array}$ & $\begin{array}{l}\text { Upper- } \\
\text { middle } \\
\text { category }\end{array}$ \\
\hline \multirow[t]{2}{*}{48} & $\begin{array}{l}\text { Fellmeth et al., } \\
2020[84]\end{array}$ & 2015-2016 & $\begin{array}{l}\text { Thai-Myanmar } \\
\text { Border }\end{array}$ & $\begin{array}{c}\text { Other non- } \\
\text { population } \\
\text { based design }\end{array}$ & Not stated & Longitudinal & $\begin{array}{l}\text { Multi-variable logistic } \\
\text { regression }\end{array}$ & $\begin{array}{l}\text { Moderate or severe } \\
\text { perinatal depression }\end{array}$ & $\begin{array}{l}\text { Upper- } \\
\text { middle } \\
\text { category }\end{array}$ \\
\hline & \multicolumn{9}{|c|}{ Multiple Countries } \\
\hline 49 & $\begin{array}{l}\text { Gibbs et al., } \\
2019 \text { [85] }\end{array}$ & $\begin{array}{c}2016-2017 \\
2017\end{array}$ & $\begin{array}{l}\text { Afghanistan } \\
\text { Palestine }\end{array}$ & $\begin{array}{c}\text { Afghanistan: } \\
\text { Intervention } \\
\text { study } \\
\text { Palestine: } \\
\text { Population- } \\
\text { based } \\
\text { design } \\
\end{array}$ & $\begin{array}{l}\text { Afghanistan: } \\
\text { WfWI } \\
\text { Palestine: not } \\
\text { stated }\end{array}$ & $\begin{array}{l}\text { Cross- } \\
\text { sectional } \\
\text { (baseline) } \\
\text { Cross- } \\
\text { sectional }\end{array}$ & $\begin{array}{l}\text { Multi-variable logistic } \\
\text { regression }\end{array}$ & $\begin{array}{l}\text { Family history of girl or } \\
\text { woman relative being } \\
\text { killed in the name of } \\
\text { honor by another family } \\
\text { member }\end{array}$ & $\begin{array}{l}\text { Upper- } \\
\text { middle } \\
\text { category }\end{array}$ \\
\hline 50 & $\begin{array}{c}\text { Misch and } \\
\text { Yount, 2014 } \\
{[86]}\end{array}$ & $\begin{array}{c}2008-2009 \\
2007 \\
2010-2011\end{array}$ & $\begin{array}{c}\text { Kenya } \\
\text { Liberia } \\
\text { Zimbabwe }^{+}\end{array}$ & $\begin{array}{l}\text { Population- } \\
\text { based } \\
\text { design }\end{array}$ & $\begin{array}{l}\text { Demographic } \\
\text { Health } \\
\text { Survey }\end{array}$ & $\begin{array}{l}\text { Cross- } \\
\text { sectional }\end{array}$ & $\begin{array}{l}\text { Multi-variable logistic } \\
\text { regression (violence } \\
\text { typologies included } \\
\text { within same model) }\end{array}$ & $\begin{array}{l}\text { Previous birth } \\
\text { breastfeeding initiation } \\
\text { 1-h after delivery } \\
\text { Past } 24-\mathrm{h} \text { exclusive } \\
\text { breastfeeding }\end{array}$ & $\begin{array}{l}\text { Upper- } \\
\text { middle } \\
\text { category }\end{array}$ \\
\hline 51 & $\begin{array}{c}\text { Gámez and } \\
\text { Speizer, 2010 } \\
\text { [87] }\end{array}$ & $\begin{array}{c}2007 \\
2007 \\
2005-2006\end{array}$ & $\begin{array}{c}\text { Liberia } \\
\text { DRC } \\
\text { Zimbabwe }^{+}\end{array}$ & $\begin{array}{l}\text { Population- } \\
\text { based } \\
\text { design }\end{array}$ & $\begin{array}{c}\text { Demographic } \\
\text { Health } \\
\text { Survey }\end{array}$ & $\begin{array}{l}\text { Cross- } \\
\text { sectional }\end{array}$ & $\begin{array}{l}\text { Multi-variable logistic } \\
\text { regression }\end{array}$ & Premarital sexual debut & $\begin{array}{l}\text { Upper- } \\
\text { middle } \\
\text { category }\end{array}$ \\
\hline
\end{tabular}

Note: When not explicitly indicated by the authors, the designation of "primary analysis model" was based on the first multivariate model provided per article. Thus, sensitivity testing was not included. If an article did not provide multivariate findings, then bivariate findings were reported; if bivariate findings were not reported, then descriptive findings were reported. ${ }^{+}$indicates that data from at least one other country was included in the article but excluded from this review; ${ }^{++}$indicates that the article includes data from men; ${ }^{+++}$indicates that two stages of analytical modeling were included for extraction; ${ }^{++++}$indicates that only non-binary measurements are indicated in parentheses. Acronyms-mo: month; HIV: Human Immunodeficiency Virus; ATI: Antiretroviral Therapy Interruption; IPV: intimate partner violence; MSM: Men who have Sex with Men; FSW: Female Sex Workers; TWG: Transgender Women; DRC: Democratic Republic of the Congo; PTSD: Post-Traumatic Stress Disorder; SDs: Standard Deviations; UIP: Unintended last Pregnancy; PMTCT: Prevention of Mother To Child Transmission; ARV: Antiretrovirals; HBsAG: Hepatitis-B Virus Surface Antigen; CQI-PMTCT: Continuous Quality Improvement for Prevention of Mother To Child Transmission; PLACE: Priorities for Local AIDS Control Efforts; WfWI: Women for Women International; RMA: Reaching Married Adolescents; IMAGES: International Men and Gender Equality Survey; GHESKIO: Haitian Group for the Study of Kaposi's Sarcoma and Opportunistic Infections; RCCS: Rakai Community Cohort Study.

\subsection{Impacts of IPV}

Consequences of IPV were identified across two primary levels: individual/survivor and microsystem/relationship (see Table 2). While all but six inferential articles demonstrated significant associations between at least one form of IPV and adverse individuallevel impacts, these findings were often related to the ill-health of the survivor. Only two articles examined how direct IPV experience was associated with future IPV victimization $(n=1)$ or perpetration $(n=1)$, while five articles included variables related to healthcare access, healthcare adherence, or health status disclosure.

Except for one article that examined IPV perpetration and one article that examined IPV revictimization, all of the individual-level associations with IPV were directly related to ill-health $(n=41)$. The most common associations were in the mental health category $(\mathrm{n}=11)$. Each of the 11 mental health studies included significant associations between IPV experience and worse mental health, with mental health variables ranging from aggregate wellness measures (including poor mental health and mental component summary) to specific conditions (including PTSD, substance abuse, and depression) or suicide variables (including ideation, thoughts, and attempts). The most common mental health variable was depression, which was included in six-articles and included perinatal depression, major depressive disorder, and past one-week, two-week, or four-week depression symptoms.

Other health categories at the individual level included the groupings of reproductive health $(n=8)$, HIV and other sexually transmitted infections $(n=7)$, pregnancy, birth, and infancy $(n=7)$, healthcare access, adherence, or disclosure $(n=5)$, overall health $(n=2)$, substance use $(2.17 \% \mathrm{n}=1)$, and fatal injuries $(\mathrm{n}=1)$. Significant findings were consistent across groupings, linking IPV with adverse physical health and mental health. Only seven of the articles included no significant results concerning individual-level impacts of IPV; moreover, there were two descriptive articles $(n=2)$ that were excluded when reviewing significance. 
Table 2. Associations with intimate partner violence, per study.

\begin{tabular}{|c|c|c|c|c|c|c|c|c|c|c|c|c|c|c|c|c|c|c|c|}
\hline \multirow{2}{*}{$\begin{array}{l}\text { Impact Categories, by Level of } \\
\text { Ecological Framework }\end{array}$} & \multicolumn{19}{|c|}{ Corresponding Article } \\
\hline & 1 & 2 & 3 & 4 & 5 & 6 & 7 & 8 & 9 & 10 & 11 & 12 & 13 & 14 & 15 & 16 & 17 & 18 & 19 \\
\hline \multicolumn{20}{|l|}{ Individual/survivor level } \\
\hline $\begin{array}{l}\text { HIV and other sexually transmitted } \\
\text { infections }\end{array}$ & - & - & - & - & - & - & - & - & - & - & - & - & - & - & - & - & - & - & - \\
\hline Pregnancy, birth, and infancy & - & - & - & - & - & - & - & - & - & S & - & - & - & - & - & S & - & - & - \\
\hline Substance use & - & - & - & - & - & - & - & - & - & - & - & - & - & - & - & - & - & - & - \\
\hline Mental health & S & - & - & $S$ & $\mathrm{~S}$ & S & - & - & - & - & - & - & - & - & - & - & - & - & \\
\hline Overall health & - & $\mathrm{N} / \mathrm{A}$ & - & - & - & - & - & - & - & - & - & - & - & - & - & - & - & - & - \\
\hline Fatal injuries & - & - & - & - & - & - & - & - & - & - & - & - & - & - & - & - & - & - & - \\
\hline Reproductive health & - & - & S & - & - & - & - & - & - & - & - & - & - & $\mathrm{S}$ & $\mathrm{S}$ & - & - & $\mathrm{S}$ & - \\
\hline Perpetration & - & - & - & - & - & - & - & - & - & - & - & - & - & - & - & - & - & - & - \\
\hline Revictimization & - & - & - & - & - & - & - & - & - & - & - & - & - & - & - & - & $\mathrm{S}$ & - & - \\
\hline $\begin{array}{l}\text { Healthcare access, adherence, or disclosure } \\
\text { Microsystem/relationship level }\end{array}$ & - & - & - & - & - & - & NS & - & - & - & $\mathrm{S}$ & - & - & - & - & - & - & - & - \\
\hline $\begin{array}{l}\text { IPV perpetration during adulthood } \\
\text { (children) }\end{array}$ & - & - & - & - & - & - & - & - & - & - & - & - & - & - & - & - & - & - & NS \\
\hline Lifetime IPV victimization (children) & - & - & - & - & - & - & - & $S$ & - & - & - & - & $S$ & - & - & - & - & - & - \\
\hline Physical health conditions (children) & - & - & - & - & - & - & - & - & - & - & - & S & - & _- & - & _- & - & _- & _- \\
\hline Emotional/behavioral problems (children) & - & - & - & - & - & - & - & - & $\mathrm{S}$ & - & - & - & - & - & - & - & - & - & - \\
\hline Accepting attitudes of IPV (children) & - & - & - & - & - & - & - & - & - & - & - & - & - & - & - & - & - & - & \\
\hline Martial disruption (partners) & - & - & - & - & - & - & - & - & - & - & - & - & - & - & - & - & - & - & - \\
\hline Fatal injuries (familial homicides) & - & - & - & - & - & - & - & - & - & - & - & - & - & - & - & - & - & - & - \\
\hline \multirow{2}{*}{$\begin{array}{l}\text { Impact Categories, by Level of } \\
\text { Ecological Framework }\end{array}$} & \multicolumn{19}{|c|}{ Corresponding Article } \\
\hline & 20 & 21 & $22^{+}$ & 23 & 24 & 25 & 26 & 28 & 29 & 30 & 31 & 32 & 33 & 34 & 35 & 36 & 37 & 38 & 39 \\
\hline Individual/survivor level & & & & & & & & & & & & & & & & & & & \\
\hline $\begin{array}{c}\text { HIV and other sexually transmitted } \\
\text { infections }\end{array}$ & - & NS & - & $\mathrm{S}$ & $\mathrm{S}$ & S & - & - & - & - & $\mathrm{S}$ & - & - & - & - & - & - & - & - \\
\hline Pregnancy, birth, and infancy & - & - & S & - & - & - & - & - & - & - & - & - & - & - & - & S & - & - & - \\
\hline Substance use & NS & - & - & - & - & - & - & - & - & - & - & - & - & - & - & - & - & - & - \\
\hline Mental health & $\mathrm{S}$ & - & - & - & - & - & $\mathrm{S}$ & - & - & - & - & - & - & & $\mathrm{S}$ & - & $\mathrm{S}$ & - & - \\
\hline Overall health & - & - & - & - & - & - & - & - & - & - & - & - & - & - & - & - & - & - & - \\
\hline Fatal injuries & - & - & - & - & - & - & - & - & - & - & - & - & N/A & - & - & - & - & - & - \\
\hline Reproductive health & - & - & - & - & - & - & - & - & - & - & - & - & - & NS & - & - & - & - & - \\
\hline Perpetration & - & - & - & - & - & - & - & - & - & - & - & - & - & - & - & - & - & $\mathrm{S}$ & - \\
\hline Revictimization & - & - & - & - & - & - & - & - & - & - & - & - & - & - & - & - & - & - & - \\
\hline $\begin{array}{l}\text { Healthcare access, adherence, or disclosure } \\
\text { Microsystem/relationship level }\end{array}$ & - & NS & - & - & - & - & - & $\mathrm{S}$ & NS & - & - & - & - & - & - & - & - & - & - \\
\hline $\begin{array}{l}\text { IPV perpetration during adulthood } \\
\text { (children) }\end{array}$ & - & - & - & - & - & - & - & - & - & - & - & - & - & - & - & - & - & $\mathrm{S}$ & $\mathrm{S}$ \\
\hline Lifetime IPV victimization (children) & - & - & S & - & - & - & - & - & - & - & - & - & - & - & - & - & - & - & - \\
\hline Emotional/behavioral problems (children) & - & - & - & - & - & - & - & - & - & - & - & - & - & - & - & - & - & - & - \\
\hline Physical health conditions (children) & - & - & - & - & - & - & - & - & - & - & - & - & - & - & - & - & - & - & - \\
\hline Accepting attitudes of IPV (children) & - & - & - & - & - & - & - & - & - & - & - & $\mathrm{S}$ & - & - & - & - & - & - & - \\
\hline Martial disruption (partners) & - & - & - & - & - & - & - & - & - & S & - & - & - & - & - & - & - & - & - \\
\hline Fatal injuries (familial homicides) & - & - & - & - & - & - & - & - & - & - & - & - & - & - & - & - & - & - & - \\
\hline \multirow{2}{*}{$\begin{array}{l}\text { Impact Categories, by Level of } \\
\text { Ecological Framework }\end{array}$} & \multicolumn{12}{|c|}{ Corresponding Article } & & & & & & & \\
\hline & 40 & 41 & 42 & 43 & 44 & 45 & 46 & 47 & 48 & 49 & 50 & 51 & & & & & & & \\
\hline Individual/survivor level & & & & & & & & & & & & & & & & & & & \\
\hline $\begin{array}{c}\text { HIV and other sexually transmitted } \\
\text { infections }\end{array}$ & S & - & S & - & - & - & - & - & - & - & - & - & & & & & & & \\
\hline Pregnancy, birth, and infancy & - & - & - & S & - & - & - & NS & - & - & S & - & & & & & & & \\
\hline Substance use & - & - & - & - & - & - & - & - & - & - & - & - & & & & & & & \\
\hline Mental health & - & - & - & - & $\mathrm{S}$ & S & - & - & S & - & - & & & & & & & & \\
\hline Overall health & - & - & - & - & $\mathrm{S}$ & - & - & - & - & - & - & - & & & & & & & \\
\hline Fatal injuries & - & - & - & - & - & - & - & - & - & - & - & - & & & & & & & \\
\hline Reproductive health & - & - & - & - & - & - & $\mathrm{S}$ & - & - & - & - & NS & & & & & & & \\
\hline Perpetration & - & - & - & - & - & - & - & - & - & - & - & - & & & & & & & \\
\hline Revictimization & - & - & - & - & - & - & - & - & - & - & - & - & & & & & & & \\
\hline $\begin{array}{l}\text { Healthcare access, adherence, or disclosure } \\
\text { Microsystem/relationship level }\end{array}$ & - & - & - & - & - & - & - & - & - & - & - & - & & & & & & & \\
\hline $\begin{array}{l}\text { IPV perpetration during adulthood } \\
\text { (children) }\end{array}$ & - & - & - & - & - & - & - & - & - & - & - & - & & & & & & & \\
\hline Lifetime IPV victimization (children) & - & S & - & - & - & - & - & - & - & - & - & - & & & & & & & \\
\hline Emotional/behavioral problems (children) & - & - & - & - & - & - & - & - & - & - & - & - & & & & & & & \\
\hline Physical health conditions (children) & - & - & - & - & - & & - & - & - & - & - & - & & & & & & & \\
\hline Accepting attitudes of IPV (children) & - & - & - & - & - & - & - & - & - & - & - & - & & & & & & & \\
\hline Martial disruption (partners) & - & - & - & - & - & - & - & - & - & - & - & - & & & & & & & \\
\hline Fatal injuries (familial homicides) & - & - & - & - & - & - & - & - & - & S & - & - & & & & & & & \\
\hline
\end{tabular}

Note: The health categories were informed by the World Health Organization's classification of health outcomes associated with intimate and non-IPV (WHO, 2013). Other categories were designated based on the authors' knowledge of violence literature and the general themes presented in the findings. Thus, the categories should only be viewed as representative of the included literature and were not meant to be an exhaustive list of potential categories associated with violence. $S$ indicates that at least one of the intimate partner violence variables included within the primary model(s) was significantly associated with a dependent variable, NS indicates that none of the intimate partner violence variables were significantly associated with a dependent variable, N/A indicates that inferential statistics were not conducted, -indicates that outcome group was not included within the analytical modeling. Significance was determined by a $p$-value of $<0.05$; if no $p$-value was provided then adjusted odds ratio were considered significant if the confidence intervals were consistently greater than or less than $1.00 .^{+}$indicates that two stages of analytical modeling were included for extraction. Acronyms: IPV-intimate partner violence; HIV—Human Immunodeficiency Virus. 
Fewer articles included impacts of IPV at the microsystem level $(23.53 \%, \mathrm{n}=12)$. The 10 articles that examined the intergenerational impact of IPV between parents or caregivers often focused on IPV perpetration $(n=3)$ or victimization $(n=4)$ during adulthood. Other categories, each examined by one article, included physical health conditions during childhood, emotional and behavioral problems during childhood, and accepting attitudes of IPV during adulthood. All of these articles included significant associations with at least one of the IPV variable(s) and an adverse outcome, except one article that did not find a significant association between childhood exposure and perpetration during adulthood. Moreover, one article examined the significant association between IPV and marital disruption. The final article, examining the associations of IPV with relationships, identified that women who experienced physical violence in the past year were more likely to report a female relative being killed in the name of honor by another family member. While community-level IPV (i.e., societal level violence) was included as an independent variable in three articles, no articles examined societal-level impacts of IPV.

\subsection{IPV Variables}

The measurement of IPV varied across articles (See Table 3), with a little less than half including more than one measure of IPV $(43.14 \%, n=22)$. However, there were some notable themes in measurement, including the tendency to focus on direct victimization of violence $(86.27 \%, \mathrm{n}=44)$. When measuring direct victimization of IPV, articles often included the timeframe of lifetime exposure for at least one of the violence variables $(n=29)$. The most common form of IPV was multiple or any form $(n=28)$, and most measures of direct victimization of IPV were binary $(n=37)$.

Table 3. Classification of intimate partner violence as independent variable(s), per study.

\begin{tabular}{|c|c|c|c|c|c|c|c|c|c|c|c|c|c|c|c|c|c|c|c|c|}
\hline \multirow{2}{*}{\multicolumn{2}{|c|}{ IPV Variable Classification, by Level }} & \multicolumn{19}{|c|}{ Corresponding Article Number } \\
\hline & & 1 & 2 & 3 & 4 & 5 & 6 & 7 & 8 & 9 & 10 & 11 & 12 & 13 & 14 & 15 & 16 & 17 & 18 & 19 \\
\hline \multicolumn{21}{|c|}{ Personal victimization of intimate partner violence } \\
\hline \multirow[t]{8}{*}{ Timeframe } & Lifetime & - & $x$ & $x$ & $x$ & $x$ & - & $x$ & - & - & - & $\mathrm{x}$ & - & - & $\mathrm{x}$ & $\mathrm{x}$ & - & - & $\mathrm{x}$ & - \\
\hline & Current partnership & - & - & - & - & - & - & - & - & - & - & - & - & - & - & - & - & - & - & - \\
\hline & Previous partnership & - & - & - & - & - & - & - & - & - & - & - & - & - & - & - & - & $x$ & - & - \\
\hline & Past 12 -mo or past year & $x$ & - & - & $x$ & - & - & - & - & - & - & - & $x$ & - & - & - & - & - & - & - \\
\hline & Past 3-months & - & - & - & - & - & $\mathrm{x}$ & - & - & - & - & - & - & - & - & - & - & - & - & - \\
\hline & Past 1 -month & - & - & - & - & $x$ & - & - & - & - & - & - & - & - & - & - & - & - & - & - \\
\hline & During pregnancy & - & - & - & - & - & - & - & - & - & $x$ & - & - & - & - & - & $x$ & - & - & - \\
\hline & Last incidence & - & - & - & - & - & - & - & - & - & - & - & - & - & - & - & - & - & - & - \\
\hline \multirow[t]{7}{*}{ Forms } & Multiple forms or any IPV & $\mathrm{x}$ & - & - & - & $\mathrm{x}$ & - & $\mathrm{x}$ & - & - & $\mathrm{x}$ & - & $\mathrm{x}$ & - & - & - & $x$ & $x$ & $x$ & - \\
\hline & AGBH & - & - & - & - & - & - & - & - & - & - & - & - & - & - & - & - & - & - & - \\
\hline & Emotional & - & - & - & - & - & $\mathrm{x}$ & - & - & - & - & - & - & - & - & - & $x$ & - & - & - \\
\hline & Physical & - & $x$ & $x$ & $x$ & - & $\mathrm{x}$ & - & - & - & - & $x$ & - & - & $x$ & $x$ & $x$ & - & - & - \\
\hline & Physiological & - & - & $x$ & $x$ & - & - & - & - & - & - & - & - & - & - & - & - & - & - & - \\
\hline & Sexual & - & - & $x$ & $x$ & - & $\mathrm{x}$ & - & - & - & - & $x$ & $x$ & - & $x$ & $x$ & $x$ & - & $x$ & - \\
\hline & Verbal & - & - & - & - & - & - & - & - & - & - & - & - & - & - & - & - & - & - & - \\
\hline \multirow[t]{5}{*}{ Measurement } & Nominal (binary) & $x$ & $x$ & $x$ & - & - & $\mathrm{x}$ & $x$ & - & - & $x$ & $x$ & - & - & $x$ & $x$ & $x$ & $x$ & $x$ & - \\
\hline & Nominal (>2 categories) & - & - & - & $x$ & - & - & - & - & - & - & - & - & - & - & - & - & - & - & - \\
\hline & Ordinal & - & - & - & - & - & - & - & - & - & - & - & - & - & - & - & - & - & - & - \\
\hline & Interval & - & - & - & - & - & - & - & - & - & - & - & - & - & - & - & - & - & - & - \\
\hline & Ratio & - & - & - & - & $\mathrm{x}$ & - & - & - & - & - & - & - & - & - & - & - & - & - & - \\
\hline \multicolumn{21}{|c|}{ Childhood witnessing of intimate partner violence between parents } \\
\hline Timeframe & During childhood & - & - & - & - & - & - & - & $\mathrm{x}$ & $\mathrm{x}$ & - & - & $\mathrm{x}$ & $\mathrm{x}$ & - & - & - & - & - & $x$ \\
\hline \multirow[t]{2}{*}{ Forms } & Multiple forms or any IPV & - & - & - & - & - & - & - & $\mathrm{x}$ & $\mathrm{x}$ & - & - & $\mathrm{x}$ & $\mathrm{x}$ & - & - & - & - & - & $\mathrm{x}$ \\
\hline & Physical & - & - & - & - & - & - & - & - & - & - & - & - & - & - & - & - & - & - & - \\
\hline \multirow[t]{2}{*}{ Measurement } & Nominal (binary) & - & - & - & - & - & - & - & $x$ & $x$ & - & - & $x$ & $x$ & - & - & - & - & - & $x$ \\
\hline & \multicolumn{20}{|c|}{ Community rates of intimate partner violence } \\
\hline \multirow[t]{2}{*}{ Timeframe } & Lifetime of respondents & - & - & - & - & - & - & - & - & - & - & - & - & - & - & - & - & - & - & - \\
\hline & Past 5-years & - & - & - & - & - & - & - & - & - & - & - & - & - & - & - & - & - & $\mathrm{x}$ & - \\
\hline \multirow[t]{3}{*}{ Forms } & Multiple forms & - & - & - & - & - & - & - & - & - & - & - & - & - & - & - & - & - & $x$ & - \\
\hline & Physical & - & - & - & - & - & - & - & - & - & - & - & - & - & - & - & - & - & - & - \\
\hline & Sexual & - & - & - & - & - & - & - & - & - & - & - & - & - & - & - & - & - & $x$ & - \\
\hline \multirow{3}{*}{ Measurement } & Nominal (binary) & - & - & - & - & - & - & - & - & - & - & - & - & - & - & - & - & - & - & - \\
\hline & Ordinal & - & - & - & - & - & - & - & - & - & - & - & - & - & - & - & - & - & - & - \\
\hline & Ratio & - & - & - & - & - & - & - & - & - & - & - & - & - & - & - & - & - & $x$ & - \\
\hline
\end{tabular}


Table 3. Cont.

\begin{tabular}{|c|c|c|c|c|c|c|c|c|c|c|c|c|c|c|c|c|c|c|c|c|c|}
\hline \multirow{2}{*}{\multicolumn{2}{|c|}{ IPV Variable Classification, by Level }} & \multicolumn{20}{|c|}{ Corresponding Article Number } \\
\hline & & 20 & 21 & 22 & 23 & 24 & 25 & 26 & 27 & 28 & 29 & $30^{+}$ & 31 & 32 & 33 & 34 & 35 & 36 & 37 & 38 & 39 \\
\hline \multicolumn{22}{|c|}{ Personal victimization of intimate partner violence } \\
\hline \multirow[t]{8}{*}{ Timeframe } & Lifetime & $\mathrm{x}$ & $\mathrm{x}$ & $\mathrm{x}$ & $\mathrm{x}$ & $\mathrm{x}$ & $\mathrm{x}$ & $\mathrm{x}$ & $\mathrm{x}$ & - & & - & $\mathrm{x}$ & - & - & - & $\mathrm{x}$ & $\mathrm{x}$ & $\mathrm{x}$ & $\mathrm{x}$ & - \\
\hline & Current partnership & - & - & - & - & - & - & - & - & $\mathrm{x}$ & - & - & - & - & - & $\mathrm{x}$ & - & - & - & - & - \\
\hline & Previous partnership & - & - & - & - & - & - & - & - & - & - & - & - & - & - & - & - & - & - & - & - \\
\hline & Past 12 -mo or past year & - & - & - & - & - & - & - & - & - & - & $\mathrm{x}$ & - & - & - & - & - & $\mathrm{x}$ & - & - & - \\
\hline & Past 3-months & - & - & - & - & - & - & - & - & - & - & - & - & - & - & - & - & - & - & - & - \\
\hline & Past 1-month & - & - & - & - & - & - & - & - & - & - & - & - & - & - & - & - & - & - & - & - \\
\hline & During pregnancy & - & - & - & - & - & - & - & - & - & - & - & - & - & - & - & - & $\mathrm{x}$ & - & - & - \\
\hline & Last incidence & - & - & - & - & - & - & - & - & - & - & - & - & - & $\mathrm{x}$ & - & - & - & - & - & - \\
\hline \multirow[t]{7}{*}{ Forms } & Multiple forms or any IPV & $\mathrm{x}$ & $\mathrm{x}$ & $\mathrm{x}$ & $\mathrm{x}$ & $\mathrm{x}$ & $\mathrm{x}$ & $\mathrm{x}$ & $\mathrm{x}$ & $\mathrm{x}$ & $\mathrm{x}$ & - & $\mathrm{x}$ & - & $\mathrm{x}$ & $\mathrm{x}$ & $\mathrm{x}$ & $\mathrm{x}$ & $\mathrm{x}$ & $\mathrm{x}$ & - \\
\hline & AGBH & - & - & - & - & $\mathrm{x}$ & - & - & - & - & - & - & - & - & - & - & - & - & - & - & - \\
\hline & Emotional & - & $\mathrm{x}$ & $\mathrm{x}$ & - & $\mathrm{x}$ & - & - & - & - & - & $\mathrm{x}$ & - & - & - & - & $\mathrm{x}$ & $\mathrm{x}$ & - & - & - \\
\hline & & - & $\mathrm{x}$ & $\mathrm{x}$ & - & $\mathrm{x}$ & - & - & - & - & - & $\mathrm{x}$ & $\mathrm{x}$ & - & - & - & $\mathrm{x}$ & $\mathrm{x}$ & - & - & - \\
\hline & Physiological & - & - & - & - & - & - & - & - & - & - & - & - & - & - & - & - & - & - & - & - \\
\hline & Sexual & - & $\mathrm{x}$ & $\mathrm{x}$ & - & $\mathrm{x}$ & - & - & - & - & - & $\mathrm{x}$ & $\mathrm{x}$ & - & - & - & $\mathrm{x}$ & $\mathrm{x}$ & - & - & \\
\hline & Ve & - & - & - & - & - & - & - & - & - & - & - & $\mathrm{x}$ & - & - & - & - & - & - & - & - \\
\hline \multirow[t]{6}{*}{ Measurement } & Nominal (binary) & $\mathrm{x}$ & $\mathrm{x}$ & $\mathrm{x}$ & $\mathrm{x}$ & $\mathrm{x}$ & - & $\mathrm{x}$ & $\mathrm{x}$ & $\mathrm{x}$ & $\mathrm{x}$ & $\mathrm{x}$ & $\mathrm{x}$ & - & $\mathrm{x}$ & $\mathrm{x}$ & - & $\mathrm{x}$ & $\mathrm{x}$ & $\mathrm{x}$ & - \\
\hline & Nominal ( $>2$ categories) & - & - & - & - & - & - & - & - & - & - & - & - & - & - & - & - & - & - & - & - \\
\hline & Ordinal & - & - & - & - & - & - & - & - & - & - & - & $\mathrm{x}$ & - & - & - & $\mathrm{x}$ & - & - & - & - \\
\hline & Interval & - & - & - & - & - & $\mathrm{x}$ & - & - & - & - & - & - & - & - & - & - & - & - & - & - \\
\hline & Ratio & & & & & & & - & & & & - & - & - & - & - & - & - & - & - & \\
\hline & \multicolumn{21}{|c|}{ Childhood witnessing of intimate partner violence between parents } \\
\hline Timeframe & Lifetime of respondents & - & - & $\mathrm{x}$ & - & - & - & - & - & - & - & - & - & $\mathrm{x}$ & - & - & - & - & - & $\mathrm{x}$ & $\mathrm{x}$ \\
\hline Forms & Multiple forms or any IPV & - & - & $\mathrm{x}$ & - & - & - & - & - & - & - & - & - & $\mathrm{x}$ & - & - & - & - & - & $\mathrm{x}$ & $\mathrm{x}$ \\
\hline & & - & - & - & - & - & - & - & - & - & - & - & - & - & - & - & - & - & - & - & \\
\hline Measurement & Nominal (binary) & - & - & $\mathrm{x}$ & - & - & - & - & - & - & - & - & - & $\mathrm{x}$ & - & - & - & - & - & $\mathrm{x}$ & $\mathrm{x}$ \\
\hline \multicolumn{22}{|c|}{ Community rates of intimate partner violence } \\
\hline Timeframe & Lifetime of respondents & - & & $\mathrm{x}$ & - & - & - & - & - & - & & - & - & - & - & - & - & - & - & - & - \\
\hline \multirow{4}{*}{ Forms } & Past 5-years & - & - & $\hat{-}$ & - & - & - & - & - & - & - & - & - & - & - & - & - & - & - & - & - \\
\hline & Multiple forms & - & - & $\mathrm{x}$ & - & - & - & - & - & - & - & - & - & - & - & - & - & - & - & - & - \\
\hline & & - & - & - & - & - & - & - & - & - & - & - & - & - & - & - & - & - & - & - & - \\
\hline & & - & - & - & - & - & - & - & - & - & - & - & - & - & - & - & - & - & - & - & - \\
\hline \multirow[t]{3}{*}{ Measurement } & Nominal (binary) & - & - & - & - & - & - & - & - & - & - & - & - & - & - & - & - & - & - & - & - \\
\hline & & - & - & $\mathrm{x}$ & - & - & - & - & - & - & - & - & - & - & - & - & - & - & - & - & - \\
\hline & Ratio & - & - & - & - & - & - & - & - & - & - & - & - & - & - & - & - & - & - & - & - \\
\hline
\end{tabular}

\begin{tabular}{|c|c|c|c|c|c|c|c|c|c|c|c|c|c|}
\hline \multirow{2}{*}{\multicolumn{2}{|c|}{ IPV Variable Classification, by Level }} & \multicolumn{12}{|c|}{ Corresponding Article Number } \\
\hline & & 40 & 41 & 42 & 43 & 44 & 45 & 46 & 47 & 48 & 49 & 50 & 51 \\
\hline \multirow{9}{*}{ Timeframe } & Personal victim & of $i$ & tima & te $\mathrm{p}$ & tne & viol & nce & & & & & & \\
\hline & Lifetime & $\mathrm{x}$ & - & - & $\mathrm{x}$ & $\mathrm{x}$ & $\mathrm{x}$ & - & $\mathrm{x}$ & $\mathrm{x}$ & - & - & - \\
\hline & Current partnership & - & - & - & - & - & - & - & - & - & - & - & - \\
\hline & Previous partnership & - & - & - & - & - & - & - & - & - & - & - & - \\
\hline & Past 12 -mo or past year & - & - & $\mathrm{x}$ & - & - & $\mathrm{x}$ & $\mathrm{x}$ & - & - & $\mathrm{x}$ & - & - \\
\hline & Past 3-months & - & - & - & - & - & - & - & - & - & - & - & - \\
\hline & Past 1-month & - & - & - & - & - & - & - & - & - & - & - & - \\
\hline & During pregnancy & - & - & - & - & - & - & - & - & - & - & $\mathrm{x}$ & - \\
\hline & Last incidence & - & - & - & - & - & - & - & - & - & - & - & - \\
\hline \multirow[t]{7}{*}{ Forms } & Multiple forms or any IPV & - & - & - & - & - & $x$ & - & $x$ & $x$ & - & - & - \\
\hline & AGBH & - & - & - & - & - & - & - & - & - & - & - & - \\
\hline & Emotional & - & - & - & - & $\mathrm{x}$ & $\mathrm{x}$ & $\mathrm{x}$ & - & - & - & $\mathrm{x}$ & - \\
\hline & Physical & $\mathrm{x}$ & - & $\mathrm{x}$ & - & $\mathrm{x}$ & $\mathrm{x}$ & $\mathrm{x}$ & - & - & $\mathrm{x}$ & $\mathrm{x}$ & - \\
\hline & Physiological & $x$ & - & - & - & - & - & - & - & - & - & - & - \\
\hline & Sexual & $x$ & - & - & $x$ & - & $x$ & $x$ & - & - & - & $x$ & - \\
\hline & Verbal & - & - & - & - & - & - & - & - & - & - & - & - \\
\hline \multirow[t]{6}{*}{ Measurement } & Nominal (binary) & $\mathrm{x}$ & - & $x$ & $\mathrm{x}$ & - & $\mathrm{x}$ & $x$ & $\mathrm{x}$ & $\mathrm{x}$ & $\mathrm{x}$ & $\mathrm{x}$ & - \\
\hline & Nominal (>2 categories) & - & - & - & - & - & - & - & - & - & - & - & - \\
\hline & Ordinal & - & - & - & - & $x$ & - & - & - & - & - & - & - \\
\hline & Interval & - & - & - & - & - & - & - & - & - & - & - & - \\
\hline & Ratio & - & - & - & - & - & - & - & - & - & - & - & - \\
\hline & Childhood witnessing o & hate & $\operatorname{artn}$ & er vi & lenc & bet & reen & pare & & & & & \\
\hline \multirow{3}{*}{$\begin{array}{c}\text { Timeframe } \\
\text { Forms }\end{array}$} & Lifetime of respondents & - & $x$ & - & - & - & - & - & - & - & - & - & - \\
\hline & Multiple forms or any IPV & - & $x$ & - & - & - & - & - & - & - & - & - & - \\
\hline & Physical & - & - & - & - & - & - & - & - & - & - & - & - \\
\hline \multirow[t]{2}{*}{ Measurement } & Nominal (binary) & - & $x$ & - & - & - & - & - & - & - & - & - & - \\
\hline & Community & f int & mate & part & er $v$ & blen & & & & & & & \\
\hline \multirow[t]{2}{*}{ Timeframe } & Lifetime of respondents & - & - & r & - & - & - & - & - & - & - & - & $\mathrm{x}$ \\
\hline & Past 5-years & - & - & - & - & - & - & - & - & - & - & - & - \\
\hline \multirow[t]{3}{*}{ Forms } & Multiple forms & - & - & - & - & - & - & - & - & - & - & - & $x$ \\
\hline & Physical & - & - & - & - & - & - & - & - & - & - & - & - \\
\hline & Sexual & - & - & - & - & - & - & - & - & - & - & - & - \\
\hline \multirow[t]{3}{*}{ Measurement } & Nominal (binary) & - & - & - & - & - & - & - & - & - & - & - & - \\
\hline & Ordinal & - & - & - & - & - & - & - & - & - & - & - & - \\
\hline & Ratio & - & - & - & - & - & - & - & - & - & - & - & $x$ \\
\hline
\end{tabular}

Note: ${ }^{+}$indicates that multiple forms of IPV were included in study but not all IPV variables were included in inferential model. Acronyms—mo: month; IPV: intimate partner violence; AGBH: aggravated bodily harm.

Fewer articles included variables that identified whether respondents witnessed IPV between their parents or primary caregivers during childhood $(19.61 \%, \mathrm{n}=10)$. However, all of those articles included binary measurement of all forms of violence. Each of the 
articles $(\mathrm{n}=3)$ that included a variable of community rates of IPV included multiple or any form of IPV; one article also included community rates of sexual violence. The timeframe used to determine community rates was either the past five-year reporting from secondary data $(n=1)$ or the aggregate lifetime experience of respondents in the sample $(n=2)$. The two measurement typologies for community violence were ordinal $(n=1)$ or ratio $(n=2)$.

\subsection{Quality Assessment}

As there are no formal AXIS scoring criteria, the authors ranked articles into four categories whereby scores of 15-20 were classified as the upper category, 10-14 as the upper-middle category, $5-9$, as the lower-middle category, and $0-4$ as the lowest category. Table 1 includes the quality assessment classification of each article and Table 4 includes the aggregate AXIS results per item. Nearly all articles met the criteria for the top 31.37\% $(n=16)$ or upper-middle $62.75 \% \%(\mathrm{n}=32)$ categories, with only three $(5.88 \%)$ articles meeting the criteria for the lower-middle category and none meeting the criteria for the lowest category. The low overall scoring of items 13 and 14, both of which pertained to non-response rates, was not surprising given that only $45.10 \%(n=23)$ of articles described measures taken to categorize non-responders (item 7). The other low score was related to the measurement of risk factors and dependent variables (item 9), whereby only $25.49 \%(n=13)$ of the articles fully described how the primary variables were derived from instruments or measurements that had been trialed, piloted, or previously published.

Table 4. AXIS quality assessment of included articles, frequency per item, and quartile distribution of final score.

\begin{tabular}{|c|c|c|}
\hline & $\mathbf{n}$ & $\%$ \\
\hline \multicolumn{3}{|l|}{ Introduction } \\
\hline 1. Were the aims/objectives of the study clear? & 51 & $100.00 \%$ \\
\hline \multicolumn{3}{|l|}{ Methods } \\
\hline 2. Was the study design appropriate for the stated aim(s)? ${ }^{+}$ & 45 & $88.24 \%$ \\
\hline 3. Was the sample size justified? & 28 & $54.90 \%$ \\
\hline 4. Was the target/reference population clearly defined? (Is it clear who the research was about?) & 51 & $100.00 \%$ \\
\hline \multicolumn{3}{|l|}{$\begin{array}{l}\text { 5. Was the sample frame taken from an appropriate population base so that it closely represented the target/reference } \\
\text { population under investigation? }\end{array}$} \\
\hline $\begin{array}{l}\text { 6. Was the selection process likely to select subjects/participants that were representative of the target/reference population } \\
\text { under investigation? }\end{array}$ & 45 & $88.24 \%$ \\
\hline \multicolumn{2}{|l|}{ 7. Were measures undertaken to address and categorize non-responders? } & $45.10 \%$ \\
\hline 7.a Were measures undertaken to categories non-responders (i.e., do the authors identify the non-response rate)? & 43 & $84.31 \%$ \\
\hline 7.b Were measures undertaken to address non-responders (i.e., do the authors identify how the non-response rate was addressed)? & 25 & $49.02 \%$ \\
\hline 8. Were the risk factor and outcome variables measured appropriately to the aims of the study? & 25 & $49.02 \%$ \\
\hline 9. Were the risk factor and outcome variables measured correctly using instruments/measurements that had been trialed, & piloted, or published previously? & $25.49 \%$ \\
\hline \multicolumn{3}{|l|}{$\begin{array}{l}\text { published previously? }\end{array}$} \\
\hline \multicolumn{3}{|l|}{$\begin{array}{l}\text { 9.b Were the outcome variables measured correctly using instruments/measurements that had been trialed, piloted, or } \\
\text { published previously? }\end{array}$} \\
\hline \multirow{2}{*}{$\begin{array}{l}\text { 10. Is it clear what was used to determined statistical significance and/or precision estimates (e.g., } p \text { values, CIs)? } \\
\text { 10.a Was sensitivity testing conducted? }\end{array}$} & 50 & $98.04 \%$ \\
\hline & 2 & $3.92 \%$ \\
\hline \multirow{2}{*}{$\begin{array}{l}\text { 11. Were the methods (including statistical methods) sufficiently described to enable them to be repeated? } \\
\text { Results }\end{array}$} & 37 & $72.55 \%$ \\
\hline & & \\
\hline 12. Were the basic data adequately described? & 49 & $96.08 \%$ \\
\hline 13. Does the response rate raise concerns about non-response bias? ${ }^{+,++}$ & 2 & $3.92 \%$ \\
\hline 14. If appropriate, was information about non-responders described? ${ }^{+}$ & 1 & $1.96 \%$ \\
\hline 15. Were the results internally consistent? & 28 & $54.90 \%$ \\
\hline 15.a Were bivariate and multivariate analyses available? ${ }^{+++}$ & 33 & $64.71 \%$ \\
\hline 16. Were the results for the analyses described in the methods, presented? & 50 & $98.04 \%$ \\
\hline \multicolumn{3}{|l|}{ Discussion } \\
\hline 17. Were the authors' discussions and conclusions justified by the results? & 47 & $92.16 \%$ \\
\hline 18. Were the limitations of the study discussed? & 49 & $96.09 \%$ \\
\hline \multicolumn{3}{|l|}{ Other } \\
\hline \multirow{2}{*}{$\begin{array}{l}\text { 19. Were there any funding sources or conflicts of interest that may affect the authors' interpretation of the results? } \\
\text { 20. Was ethical approval or consent of participants attained? }\end{array}$} & 23 & $45.10 \%$ \\
\hline & 35 & $68.63 \%$ \\
\hline
\end{tabular}

Note: Quartile distribution was as follows: $31.37 \%(\mathrm{n}=16)$ top category, $62.75 \%(\mathrm{n}=32)$ upper-middle category, $5.88 \%(\mathrm{n}=3)$ lower-middle category, $0.00 \%(\mathrm{n}=0)$ lowest category. ${ }^{+}$indicates an item where only one reviewer's votes were applied; ${ }^{++}$indicates that consistent "No" voting were applied to these articles; ${ }^{+++}$descriptive studies were not included in the $\%$ calculation $(n=49)$. Acronyms-IPV: intimate partner violence. Notations- $\%$ : percentage of included studies; n: number of studies. For AXIS items that had two separate components (e.g., items 7 and 9), reviewers voted for each component separately; only articles that reviewed affirmative votes for both components by both reviewers were counted for the corresponding item. Information it italics represents additional considerations, beyond the AXIS designations. While not impacting the final score, two additional sub-items were included to determine the frequency that articles included sensitivity testing (10.a) or provided both bivariate and multivariate results (15.a). 
The averages of the first four domains in the AXIS tool—introduction, methods, results, discussion-were relatively similar; however, the final domain of "other" included two items with low averages. The first item of the "other" domain asked about funding sources or conflicts of interest that may affect the authors' interpretation of the results, whereby only half of the articles $(n=28)$ did not have overt conflicts. It is important to note that the "don't know" option was selected whenever funding information was not provided; thus, those votes did not contribute to the final count. Similarly, only $68.63 \%(n=35)$ of the articles reported ethical approval; however, this only reflects ethical approval reported within the articles. Some studies may have acquired ethical approval, but the authors did not report it. In both instances, these findings are important in regard to flagging the inconsistent reporting of ethical information.

\section{Discussion}

This review endeavored to identify the common and unique impacts of IPV in humanitarian settings across the entire ecological framework; however, no included article examined the impacts of IPV at the exosystem or macrosystem levels. Thus, we synthesized the existing empirical evidence at the individual and microsystem levels. Our synthesis corroborated previous reviews that indicated IPV to be associated with adverse physical and mental health outcomes for survivors at the individual level [10-13] and identified that IPV in humanitarian settings has detrimental impacts on family members at the microsystem level. Critically, the included articles for this review did not elucidate humanitarian-unique impacts of IPV. This is concerning, as there are many characteristics germane to humanitarian settings that could both exacerbate the prevalence of IPV [6] and magnify the impact of IPV $[9,88]$. Without a comprehensive understanding of IPV's influence across the ecological framework, important considerations for programming and policy may be overlooked and funding may continue to underserve women and girls in humanitarian settings [89]. As the evidence-base expands, we advocate for future efforts to consider how the ecological framework proposed in Figure 1 may be refined to comprehensively reflect the impacts of IPV in humanitarian settings. Few of the hypothesized impacts of IPV from Figure 1 were explored in the included articles and no novel impacts were included within the study designs of the reviewed literature, thus, highlighting huge and important data gaps.

A striking challenge with examining the included research to ascertain the impacts of IPV during humanitarian crises was the reliance on lifetime experience of IPV as a single binary variable. In addition to reflecting an oversimplification or potential measurement issue, this lifetime experience of IPV could have occurred outside the period when a context would be considered a humanitarian setting. Only ten articles included IPV experienced within the past 1 to 12 months, but it is important to flag that all of them reported a significant association between IPV and the impacts of IPV. The number of selected articles was too small to rigorously compare but this finding highlights that IPV experienced during a humanitarian crisis may be more consistently associated with adverse IPV impacts than lifetime exposure. Future research should include explicit consideration regarding how different experiences of violence before, during, and after a humanitarian crisis may uniquely influence survivors, families, and communities. Research should also explore the interactions of IPV impacts between levels of ecological framework. Such research would begin to elucidate how and to what extent various levels of the ecological framework have interactive and reinforcing influence on the impacts of IPV (also known as reciprocal determinism). This type of interconnected thinking could bolster program efficiency by supporting more targeted programming to address the most acutely influential impacts of IPV $[90,91]$.

When examining the quality assessment results, the AXIS tool yielded favorable scores for the included articles; however, there are still important gaps regarding the broader scientific evidence available. The choice of the AXIS tool was, in part, due to the crosssectional nature of most articles. Only five of included articles analyzed longitudinal data, aligning with other reviews in non-humanitarian settings with a similar dearth in 
longitudinal data $[12,13]$. Longitudinal studies from humanitarian settings are needed to temporally understand the interplay of IPV experience, humanitarian conflict/emergency, and outcomes across the ecological model. Moreover, humanitarian IPV research is geographically stymied. Of the 48 countries that received either CAP or HRP funding between 2005 and 2020 , data from only 18 countries $(37.50 \%)$ were included within a publication that met our inclusion criteria. The vast majority of data were collected within the African continent, and there were no articles from South and Central America. The included studies were also limited in their ability to consider the temporal and geographical severity of humanitarian crises. Emergency designation (Level 1-Level 3) is available for current and recent crises [92], but there is no open-source designation of crisis severity between settings and across time. The INFORM Global Crisis Severity Index (GCSI) provides as an emerging initiative that may support examination of humanitarian crisis designation and IPV impacts for future research [93]. By integrating multi-level modeling techniques of GCSI designations or other secondary data sources like Armed Conflict Location and Event Data Project (ACLED) or Uppsala Conflict Data Program (UCDP) [4,54], researchers could examine the influence of various forms of IPV between crisis severity. In the meantime, researchers should consider operationalizing humanitarian stressors, such as self-reported conflict exposure and forced migration. Researchers and donors, alike, need to consider how to more inclusively and comprehensively fix these data gaps as they impede the ability to address the impacts of IPV in humanitarian settings.

\section{Limitations}

Limitations of this systematic review should be carefully considered. The overwhelmingly significant associations demonstrated in the findings may be a result of publication biases for articles that demonstrate significant findings. Thus, we cannot exhaustively state that other dependent variables have not been examined, only that other dependent variables were not available in the published peer-reviewed literature. Future research should examine and report on a range of outcomes that have been theorized to have associations with IPV. When including common impact variables, future research should build upon existing modelling to support comparability as the misalignment in measurement across studies hindered the possibility of meta-analysis for this systematic review. Finally, this systematic review utilized the AXIS tool for quality assessment despite not all articles using cross-sectional data. While the AXIS tool was developed for cross-sectional studies, each item within the AXIS tool was relevant for longitudinal research and the authors felt it was important to apply the same criteria for each article to enable comparability.

\section{Conclusions}

IPV in humanitarian settings is pervasive, and findings from this review indicated that its impacts are far-reaching. We demonstrated that IPV was significantly associated with a range of adverse health and non-health impacts for individuals and family members. However, there remains a paucity of inclusive research examining the novel impacts of IPV in humanitarian settings across the ecological framework. An understanding of the health and non-health impacts of IPV among survivors and, importantly, their families and communities, is critical for programming to thwart the widespread harms of IPV. With any hopes of achieving the Sustainable Development Goal of Gender Equality by 2030 [94], research and investment needs to prioritize the unique experiences of IPV by survivors in humanitarian settings and the impacts across the ecological framework.

Author Contributions: The manuscript was conceptualized by L.S. and M.M. Article review and data extraction was conducted by K.T., M.M. and S.M. and supported by C.P., I.S., L.S. and L.V. Initial literature review was conducted by K.T., L.V., M.M. and S.M., M.M., C.P., I.S., K.T., L.S., L.V. and S.M. were involved in original draft writing, editing, and final approval. All authors have read and agreed to the published version of the manuscript. 
Funding: This study is made possible by the support of the American People through the US State Department Bureau of Population, Refugees and Migration. The findings of this study are the sole responsibility of the contributing authors and do not necessarily reflect the views of the United States Government.

Institutional Review Board Statement: Not applicable.

Informed Consent Statement: Not applicable.

Data Availability Statement: Not applicable.

Conflicts of Interest: The authors declare no conflict of interest.

\section{References}

1. IASC. Guidelines for Integrating Gender-Based Violence Interventions in Humanitarian Action; IASC: Geneva, Switzerland, 2015.

2. UNFPA. Managing Gender-based Violence Programmes in Emergencies. Available online: https://extranet.unfpa.org/Apps/ GBVinEmergencies/index.html (accessed on 17 December 2020).

3. Catani, C. War in the Home- an Overview of the Connection between War Traumatization and Domestic Violence. Behav. Ther. 2010, 20, 19-27. [CrossRef]

4. Kelly, J.T.D.; Colantuoni, E.; Robinson, C.; Decker, M.R. From the Battlefield to the Bedroom: A Multilevel Analysis of the Links between Political Conflict and Intimate Partner Violence in Liberia. BMJ Glob. Health 2018, 3, 668. [CrossRef]

5. Parcesepe, A.; Stark, L.; Roberts, L.; Boothby, N. Measuring Physical Violence and Rape against Somali Women Using the Neighborhood Method. Violence Women 2016, 22, 798-816. [CrossRef]

6. Stark, L.; Ager, A. A Systematic Review of Prevalence Studies of Gender-Based Violence in Complex Emergencies. Trauma Violence Abus. 2011, 127-134. [CrossRef] [PubMed]

7. Stark, L.; Warner, A.; Lehmann, H.; Boothby, N.; Ager, A. Measuring the Incidence and Reporting of Violence against Women and Girls in Liberia Using the "Neighborhood Method". Confl. Health 2013, 7, 20. [CrossRef]

8. Rubenstein, B.L.; Lu, L.Z.N.; MacFarlane, M.; Stark, L. Predictors of Interpersonal Violence in the Household in Humanitarian Settings: A Systematic Review. Trauma Violence Abus. 2020, 31-44. [CrossRef]

9. Stark, L.; Seff, I.; Reis, C. Gender-Based Violence against Adolescent Girls in Humanitarian Settings: A Review of the Evidence. Lancet Child Adolesc. Health 2020, 5, 210-222. [CrossRef]

10. Coker, A.L.; Davis, K.E.; Arias, I.; Desai, S.; Sanderson, M.; Brandt, H.M.; Smith, P.H. Physical and Mental Health Effects of Intimate Partner Violence for Men and Women. Am. J. Prev. Med. 2002, 23, 260-268. [CrossRef]

11. Campbell, J.C. Health Consequences of Intimate Partner Violence. Lancet 2002, 359, 1331-1336. [CrossRef]

12. Grose, R.G.; Roof, K.A.; Semenza, D.C.; Leroux, X.; Yount, K.M. Mental Health, Empowerment, and Violence against Young Women in Lower-Income Countries: A Review of Reviews. Aggress. Violent Behav. 2019, 46, 25-36. [CrossRef]

13. Grose, R.G.; Chen, J.S.; Roof, K.A.; Rachel, S.; Yount, K.M. Sexual and Reproductive Health Outcomes of Violence Against Women and Girls in Lower-Income Countries: A Review of Reviews. J. Sex Res. 2020, 1-20. [CrossRef] [PubMed]

14. Classen, C.C.; Palesh, O.G.; Aggarwal, R. Sexual Revictimization. Trauma Violence Abus. 2005, 6, 103-129. [CrossRef]

15. Logie, C.H.; Okumu, M.; Mwima, S.; Hakiza, R.; Irungi, K.P.; Kyambadde, P.; Kironde, E.; Narasimhan, M. Social Ecological Factors Associated with Experiencing Violence among Urban Refugee and Displaced Adolescent Girls and Young Women in Informal Settlements in Kampala, Uganda: A Cross-Sectional Study. Confl. Health 2019, 13, 60. [CrossRef]

16. Kohli, A.; Perrin, N.; Mpanano, R.M.; Banywesize, L.; Mirindi, A.B.; Banywesize, J.H.; Mitima, C.M.; Binkurhorhwa, A.K.; Bufole, N.M.; Glass, N. Family and Community Driven Response to Intimate Partner Violence in Post-Conflict Settings. Soc. Sci. Med. 2015, 146, 276-284. [CrossRef]

17. Panter-Brick, C. Conflict, Violence, and Health: Setting a New Interdisciplinary Agenda. Soc. Sci. Med. 2010, 1-6. [CrossRef]

18. Rios-Avila, F.; Canavire-Bacarreza, G.J. The Effect of Intimate Partner Violence on Labor Market Decisions: Evidence from a Multi-Ethnic Country. Int. J. Soc. Econ. 2017, 44, 75-92. [CrossRef]

19. Swanberg, J.E.; Logan, T.K.; Macke, C. Intimate Partner Violence, Employment, and The Workplace: Consequences and Future Directions. Trauma Violence Abus. 2005, 6, 286-312. [CrossRef]

20. Dalal, K.; Dawad, S. Economic Costs of Domestic Violence: A Community Study in South Africa. HealthMED 2011, 5, 1931-1940.

21. Lince-Deroche, N.; Berry, K.M.; Hendrickson, C.; Sineke, T.; Kgowedi, S.; Mulongo, M. Women's Costs for Accessing Comprehensive Sexual and Reproductive Health Services: Findings from an Observational Study in Johannesburg, South Africa. Reprod. Health 2019, 16, 179. [CrossRef]

22. Abramsky, T.; Watts, C.H.; Garcia-Moreno, C.; Devries, K.; Kiss, L.; Ellsberg, M.; Jansen, H.A.; Heise, L. What Factors Are Associated with Recent Intimate Partner Violence? Findings from the WHO Multi-Country Study on Women's Health and Domestic Violence. BMC Public Health 2011, 11, 109. [CrossRef]

23. Fulu, E.; Miedema, S.; Roselli, T.; McCook, S.; Chan, K.L.; Haardörfer, R.; Jewkes, R.; Fulu, E.; Jewkes, R.; Warner, X.; et al. Pathways between Childhood Trauma, Intimate Partner Violence, and Harsh Parenting: Findings from the UN Multi-Country Study on Men and Violence in Asia and the Pacific. Lancet Glob. Health 2017, 5, e512-e522. [CrossRef]

24. Jewkes, R. Intimate Partner Violence: Causes and Prevention. Lancet Lond. Engl. 2002, 359, 1423-1429. [CrossRef] 
25. Krug, E.G.; Mercy, J.A.; Dahlberg, L.L.; Zwi, A.B. The World Report on Violence and Health. Lancet 2002, 360, 1083-1088. [CrossRef]

26. United Nations. United Nations Security Council Resolution 1325 on Women, Peace and Security Understanding the Implications, Fulfilling the Obligations; United Nations: New York, NY, USA, 2000.

27. Pinheiro, P.S. World Report on Violence against Children; UN: Geneva, Switzerland, 2009.

28. Bundhamcharoen, K.; Odton, P.; Mugem, S.; Phulkerd, S.; Dhisayathikom, K.; Brown, D.W.; Tangcharoensathien, V. Costs of Injuries Due to Interpersonal and Self-Directed Violence in Thailand. J. Med. Assoc. Thai. 2005, 91, S110-S118.

29. Raghavendra, S.; Duvvury, N.; Ashe, S. The Macroeconomic Loss Due to Violence against Women: The Case of Vietnam. Fem. Econ. 2017, 23, 62-89. [CrossRef]

30. Roldós, M.I.; Corso, P. The Economic Burden of Intimate Partner Violence in Ecuador: Setting the Agenda for Future Research and Violence Prevention Policies. West. J. Emerg. Med. 2013, 14, 347-353. [CrossRef]

31. Heise, L.L. Violence against Women: An Integrated, Ecological Framework. Violence Woman 1998, 4, 262-290. [CrossRef]

32. Cislaghi, B.; Heise, L. Using Social Norms Theory for Health Promotion in Low-Income Countries. Health Promot. Int. 2019, 34, 616-623. [CrossRef]

33. Moher, D.; Liberati, A.; Tetzlaff, J.; Altman, D.G. Preferred Reporting Items for Systematic Reviews and Meta-Analyses: The PRISMA Statement. BMJ 2009, 332-336. [CrossRef]

34. WHO. WHO Multi-Country Study on Women's Health and Domestic Violence against Women; World Health Organization: Geneva, Switzerland, 2005.

35. United Nations Office for the Coordination of Humanitarian Affairs. Financial Tracking System. Available online: https: / / fts.unocha.org/ (accessed on 17 December 2020).

36. Downes, M.J.; Brennan, M.L.; Williams, H.C.; Dean, R.S. Development of a Critical Appraisal Tool to Assess the Quality of Cross-Sectional Studies (AXIS). BMJ Open 2016, 6, e011458. [CrossRef]

37. Goessmann, K.; Ibrahim, H.; Neuner, F. Association of War-Related and Gender-Based Violence With Mental Health States of Yazidi Women. JAMA Netw. Open 2020, 3, e2013418. [CrossRef] [PubMed]

38. Al-Atrushi, H.H.; Al-Tawil, N.G.; Shabila, N.P.; Al-Hadithi, T.S. Intimate Partner Violence against Women in the Erbil City of the Kurdistan Region, Iraq. BMC Womens Health 2013, 13, 37. [CrossRef] [PubMed]

39. Zakar, R.; Zakar, M.Z.; Mikolajczyk, R.; Krämer, A. Intimate Partner Violence and Its Association with Women's Reproductive Health in Pakistan. Int. J. Gynaecol. Obstet. 2012, 117, 1-14. [CrossRef] [PubMed]

40. Zakar, R.; Zakar, M.Z.; Mikolajczyk, R.; Kraemer, A. Spousal Violence against Women and Its Association with Women's Mental Health in Pakistan. Health Care Women Int. 2013, 34, 795-813. [CrossRef]

41. Sousa, C.; Yacoubian, K.; Flaherty Fischette, P.; Haj-Yahia, M.M. The Co-Occurrence and Unique Mental Health Effects of Political Violence and Intimate Partner Violence. J. Interpers. Violence 2018, 33, 268-292. [CrossRef]

42. Falb, K.L.; Blackwell, A.; Stennes, J.; Hussein, M.; Annan, J. Depressive Symptoms among Women in Raqqa Governorate, Syria: Associations with Intimate Partner Violence, Food Insecurity, and Perceived Needs. Glob. Ment. Health Camb. Engl. 2019,6 , e22. [CrossRef]

43. Fiorentino, M.; Sagaon-Teyssier, L.; Ndiaye, K.; Suzan-Monti, M.; Mengue, M.-T.; Vidal, L.; Kuaban, C.; March, L.; Laurent, C.; Spire, B.; et al. Intimate Partner Violence against HIV-Positive Cameroonian Women: Prevalence, Associated Factors and Relationship with Antiretroviral Therapy Discontinuity-Results from the ANRS-12288 EVOLCam Survey. Womens Health 2019, 15, 1-12. [CrossRef]

44. Peltzer, K.; Pengpid, S. Female Genital Mutilation and Intimate Partner Violence in the Ivory Coast. BMC Womens Health 2014, 14, 13. [CrossRef]

45. Alenko, A.; Girma, S.; Abera, M.; Workicho, A. Children Emotional and Behavioural Problems and Its Association with Maternal Depression in Jimma Town, Southwest Ethiopia. Gen. Psychiatry 2020, 33. [CrossRef]

46. Gebreslasie, K.Z.; Weldemariam, S.; Gebre, G.; Mehari, M.-A. Intimate Partner Violence during Pregnancy and Risk of Still Birth in Hospitals of Tigray Region Ethiopia. Ital. J. Pediatr. 2020, 46, 107. [CrossRef]

47. Sipsma, H.; Callands, T.A.; Bradley, E.; Harris, B.; Johnson, B.; Hansen, N.B. Healthcare Utilisation and Empowerment among Women in Liberia. J. Epidemiol. Community Health 2013, 67, 953-959. [CrossRef] [PubMed]

48. Sobkoviak, R.M.; Yount, K.M.; Halim, N. Domestic Violence and Child Nutrition in Liberia. Soc. Sci. Med. 2012, 74, $103-111$. [CrossRef] [PubMed]

49. Hayes, B.; van Baak, C. Risk Factors of Physical and Sexual Abuse for Women in Mali: Findings from a Nationally Representative Sample. Violence Women 2017, 23, 1361-1381. [CrossRef] [PubMed]

50. DeLong, S.M.; Brooks, M.I.; Aliou, S.; Lundgren, R.; Corneliess, C.; Johns, N.E.; Challa, S.; Carter, N.; Lauro, G.; Silverman, J.G. Married Very Young Adolescent Girls in Niger at Greatest Risk of Lifetime Male Partner Reproductive Coercion and Sexual Violence. PLoS ONE 2020, 15, 30231392. [CrossRef]

51. Silverman, J.G.; Challa, S.; Boyce, S.C.; Averbach, S.; Raj, A. Associations of Reproductive Coercion and Intimate Partner Violence with Overt and Covert Family Planning Use among Married Adolescent Girls in Niger. EClinicalMedicine 2020, $22,100393$. [CrossRef] [PubMed] 
52. Kana, M.A.; Safiyan, H.; Yusuf, H.E.; Musa, A.S.M.; Richards-Barber, M.; Harmon, Q.E.; London, S.J. Association of Intimate Partner Violence during Pregnancy and Birth Weight among Term Births: A Cross-Sectional Study in Kaduna, Northwestern Nigeria. BMJ Open 2020, 10. [CrossRef] [PubMed]

53. Abiodun, O.; Sodeinde, K.; Bamidele, F.; Ojinni, Y.; Adekeye, J.; Ohiaogu, F.; Mbonu, F. Intimate Partner Violence among Women Accessing HIV-Care Services at Tertiary Hospitals in Ogun State, Nigeria: Implications for Policy and Practice. J. Interpers. Violence 2020, 1-21. [CrossRef]

54. Kidman, R.; Palermo, T.; Bertrand, J. Intimate Partner Violence, Modern Contraceptive Use and Conflict in the Democratic Republic of the Congo. Soc. Sci. Med. 2015, 133, 2-10. [CrossRef]

55. Fleming, P.J.; McCleary-Sills, J.; Morton, M.; Levtov, R.; Heilman, B.; Barker, G. Risk Factors for Men's Lifetime Perpetration of Physical Violence against Intimate Partners: Results from the International Men and Gender Equality Survey (IMAGES) in Eight Countries. PLoS ONE 2015, 10, e0118639. [CrossRef]

56. Johnson, K.; Scott, J.; Rughita, B.; Kisielewski, M.; Asher, J.; Ong, R.; Lawry, L. Association of Sexual Violence and Human Rights Violations with Physical and Mental Health in Territories of the Eastern Democratic Republic of the Congo. JAMA J. Am. Med. Assoc. 2010, 304, 553-562. [CrossRef]

57. Gichane, M.W.; Moracco, K.E.; Thirumurthy, H.; Okitolonda, E.W.; Behets, F.; Yotebieng, M. Intimate Partner Violence and Prevention of Mother to Child Transmission of HIV: Evidence from Kinshasa, Democratic Republic of Congo. PLoS ONE 2018, 13, e0203471. [CrossRef]

58. Tiruneh, F.N.; Chuang, K.Y.; Ntenda, P.A.M.; Chuang, Y.C. Unwanted Pregnancy, Pregnancy Loss, and Other Risk Factors for Intimate Partner Violence in the Democratic Republic of the Congo. Women Health 2018, 58, 983-1000. [CrossRef]

59. Mpody, C.; Thompson, P.; Tabala, M.; Ravelomanana, N.L.R.; Malongo, F.; Kawende, B.; Behets, F.; Okitolonda, E.; Yotebieng, M. Hepatitis B Infection among Pregnant and Post-Partum Women Living with HIV and on Antiretroviral Therapy in Kinshasa, DR Congo: A Cross-Sectional Study. PLoS ONE 2019, 14, e0216293. [CrossRef]

60. Onsomu, E.O.; Abuya, B.A.; Okech, I.N.; Rosen, D.L.; Duren-Winfield, V.; Simmons, A.C. Association between Domestic Violence and HIV Serostatus among Married and Formerly Married Women in Kenya. Health Care Women Int. 2015, 36, 205-228. [CrossRef]

61. Shi, C.; Kouyoumdjian, F.G.; Dushoff, J. Intimate Partner Violence Is Associated with HIV Infection in Women in Kenya: A Cross-Sectional Analysis. BMC Public Health 2013, 13, 512. [CrossRef]

62. Seff, I.; Stark, L. A Sex-Disaggregated Analysis of How Emotional Violence Relates to Suicide Ideation in Low- and Middle-Income Countries. Child Abus. Negl. 2019, 93, 222-227. [CrossRef]

63. Phillips-Howard, P.A.; Otieno, G.; Burmen, B.; Otieno, F.; Odongo, F.; Odour, C.; Nyothach, E.; Amek, N.; Zielinski-Gutierrez, E.; Odhiambo, F.; et al. Menstrual Needs and Associations with Sexual and Reproductive Risks in Rural Kenyan Females: A Cross-Sectional Sehavioral Survey Linked with HIV Prevalence. J. Womens Health 2015, 24, 801-811. [CrossRef]

64. Kinuthia, J.; Singa, B.; McGrath, C.J.; Odeny, B.; Langat, A.; Katana, A.; Ng'ang'a, L.; Pintye, J.; John-Stewart, G. Prevalence and Correlates of Non-Disclosure of Maternal HIV Status to Male Partners: A National Survey in Kenya. BMC Public Health 2018, 18, 671. [CrossRef] [PubMed]

65. Goyette, M.; Mutiti, P.; Bukusi, D.; Wamuti, B.M.; Otieno, F.A.; Cherutich, P.; Golden, M.R.; Spiegel, H.; Richardson, B.A.; Ng'ang'a, A.; et al. HIV Assisted Partner Services among Those with and without a History of Intimate Partner Violence in Kenya. J. Acquir. Immune Defic. Syndr. 2018, 78, 16-19. [CrossRef] [PubMed]

66. Wagman, J.A.; Charvat, B.; Thoma, M.E.; Ndyanabo, A.; Nalugoda, F.; Ssekasanvu, J.; Kigozi, G.; Serwadda, D.; Kagaayi, J.; Wawer, M.J.; et al. Intimate Partner Violence as a Predictor of Marital Disruption in Rural Rakai, Uganda: A Longitudinal Study. Int. J. Public Health 2016, 61, 961-970. [CrossRef] [PubMed]

67. Kouyoumdjian, F.G.; Calzavara, L.M.; Bondy, S.J.; O'Campo, P.; Serwadda, D.; Nalugoda, F.; Kagaayi, J.; Kigozi, G.; Wawer, M.; Gray, R. Intimate Partner Violence Is Associated with Incident HIV Infection in Women in Uganda. AIDS Lond. Engl. 2013, 27, 1331-1338. [CrossRef] [PubMed]

68. Speizer, I.S. Intimate Partner Violence Attitudes and Experience among Women and Men in Uganda. J. Interpers. Violence 2010, 25, 1224-1241. [CrossRef] [PubMed]

69. Macucha, C.M.; Taunde, S.A. Domestic Homicide in Maputo Province, Mozambique. Forensic Sci. Int. Online 2020, 2, 157-161. [CrossRef]

70. Adjiwanou, V.; N’Bouke, A. Exploring the Paradox of Intimate Partner Violence and Increased Contraceptive Use in Sub-Saharan Africa. Stud. Fam. Plann. 2015, 46, 127-142. [CrossRef]

71. Shamu, S.; Zarowsky, C.; Roelens, K.; Temmerman, M.; Abrahams, N. High-Frequency Intimate Partner Violence during Pregnancy, Postnatal Depression and Suicidal Tendencies in Harare, Zimbabwe. Gen. Hosp. Psychiatry 2016, 38, 109-114. [CrossRef]

72. Shamu, S.; Munjanja, S.; Zarowsky, C.; Shamu, P.; Temmerman, M.; Abrahams, N. Intimate Partner Violence, Forced First Sex and Adverse Pregnancy Outcomes in a Sample of Zimbabwean Women Accessing Maternal and Child Health Care. BMC Public Health 2018, 18, 595. [CrossRef] [PubMed]

73. Nyamukoho, E.; Mangezi, W.; Marimbe, B.; Verhey, R.; Chibanda, D. Depression among HIV Positive Pregnant Women in Zimbabwe: A Primary Health Care Based Cross-Sectional Study. BMC Pregnancy Childbirth 2019, 19, 53. [CrossRef]

74. Kayibanda, J.F.; Alary, M. Trends and Risk Factors Associated with the Perpetration of Physical Intimate Partner Violence by Women in Haiti, 2000-2012. Women Health 2020, 60, 12-25. [CrossRef] [PubMed] 
75. Gage, A.J. Exposure to Spousal Violence in the Family, Attitudes and Dating Violence Perpetration among High School Students in Port-Au-Prince. J. Interpers. Violence 2016, 31, 2445-2474. [CrossRef]

76. Saxena, A.; Deschamps, M.; Dorvil, N.; Christophe, I.; Rosenberg, R.; Jean-Gilles, M.; Koenig, S.; Pape, J.W.; Dévieux, J.G. Association between Intimate Partner Violence and HIV Status among Haitian Women. Glob. Public Health 2019, 14, 1557-1568. [CrossRef] [PubMed]

77. Occean, J.R.; Thomas, N.; Lim, A.C.; Lovett, S.M.; Michael-Asalu, A.; Salinas-Miranda, A.A. Prevalence and Factors Associated with Intimate Partner Violence among Women in Haiti: Understanding Household, Individual, Partner, and Relationship Characteristics. J. Interpers. Violence 2020, 1-29. [CrossRef]

78. Zalla, L.C.; Herce, M.E.; Edwards, J.K.; Michel, J.; Weir, S.S. The Burden of HIV among Female Sex Workers, Men Who Have Sex with Men and Transgender Women in Haiti: Results from the 2016 Priorities for Local AIDS Control Efforts (PLACE) Study. J. Int. AIDS Soc. 2019, 22, e25281. [CrossRef]

79. Bellizzi, S.; Nivoli, A.; Salaris, P.; Ronzoni, A.R.; Pichierri, G.; Palestra, F.; Wazway, O.; Luque-Fernandez, M.A. Sexual Violence and Eclampsia: Analysis of Data from Demographic and Health Surveys from Seven Low-and Middle-Income Countries. J. Glob. Health 2019, 9, 020434. [CrossRef]

80. Gibbs, A.; Corboz, J.; Jewkes, R. Factors Associated with Recent Intimate Partner Violence Experience amongst Currently Married Women in Afghanistan and Health Impacts of IPV: A Cross Sectional Study. BMC Public Health 2018, 18, 593. [CrossRef]

81. Aye, W.T.; Lien, L.; Stigum, H.; Schei, B.; Sundby, J.; Bjertness, E. Domestic Violence Victimisation and Its Association with Mental Distress: A Cross-Sectional Study of the Yangon Region, Myanmar. BMJ Open 2020, 10, e037936. [CrossRef]

82. Tsai, L.C.; Cappa, C.; Petrowski, N. The Relationship between Intimate Partner Violence and Family Planning among Girls and Young Women in the Philippines. Glob. J. Health Sci. 2016, 8, 121-131. [CrossRef]

83. Falb, K.L.; McCormick, M.C.; Hemenway, D.; Anfinson, K.; Silverman, J.G. Symptoms Associated with Pregnancy Complications along the Thai-Burma Border: The Role of Conflict Violence and Intimate Partner Violence. Matern. Child Health J. 2014, 18, 29-37. [CrossRef]

84. Fellmeth, G.; Plugge, E.; Fazel, M.; Oo, M.M.; Pimanpanarak, M.; Phichitpadungtham, Y.; Wai, K.; Charunwatthana, P.; Simpson, J.A.; Nosten, F.; et al. Prevalence and Determinants of Perinatal Depression among Labour Migrant and Refugee Women on the Thai-Myanmar Border: A Cohort Study. BMC Psychiatry 2020, 20, 168. [CrossRef]

85. Gibbs, A.; Said, N.; Corboz, J.; Jewkes, R. Factors Associated with 'Honour Killing' in Afghanistan and the Occupied Palestinian Territories: Two Cross-Sectional Studies. PLoS ONE 2019, 14, e0219125. [CrossRef]

86. Misch, E.S.; Yount, K.M. Intimate Partner Violence and Breastfeeding in Africa. Matern. Child Health J. 2014, 18, 688-697. [CrossRef]

87. Gámez, A.M.; Speizer, I.S. Community-Level Intimate Partner Violence and the Circumstances of First Sex among Young Women from Five African Countries. Reprod. Health 2010, 7, 11. [CrossRef]

88. Bermudez, L.G.; Stark, L.; Bennouna, C.; Jensen, C.; Potts, A.; Kaloga, I.F.; Tilus, R.; Buteau, J.E.; Marsh, M.; Hoover, A.; et al. Converging Drivers of Interpersonal Violence: Findings from a Qualitative Study in Post-Hurricane Haiti. Child Abuse Negl. 2019, 89, 178-191. [CrossRef]

89. Marsh, M.; Blake, M. Where Is the Money? How the Humanitarian System Is Failing in Its Commitments to End Violence against Women and Girls; IRC. 2019. Available online: https://www.rescue.org/report/wheres-money-how-humanitarian-system-failing-fundend-violence-against-women-and-girls (accessed on 10 June 2019).

90. Schölmerich, V.L.N.; Kawachi, I. Translating the Socio-Ecological Perspective into Multilevel Interventions: Gaps between Theory and Practice. Health Educ. Behav. 2016, 43, 17-20. [CrossRef] [PubMed]

91. Golden, S.D.; McLeroy, K.R.; Green, L.W.; Earp, J.A.L.; Lieberman, L.D. Upending the Social Ecological Model to Guide Health Promotion Efforts toward Policy and Environmental Change. Health Educ. Behav. 2015, 42 (Suppl. 1), 8S-14S. [CrossRef] [PubMed]

92. IASC. IASC System-Wide Response Activations and Deactivations. Available online: https://interagencystandingcommittee. org/iasc-transformative-agenda/iasc-system-wide-response-activations-and-deactivations (accessed on 21 December 2020).

93. ACAPS. INFORM Global Crisis Severity Index: Guidance Note; ACAPS: Geneva, Switzerland, 2020.

94. UNGA. Transforming Our World: The 2030 Agenda for Sustainable Development; UNGA: New York, NY, USA, 2015. 